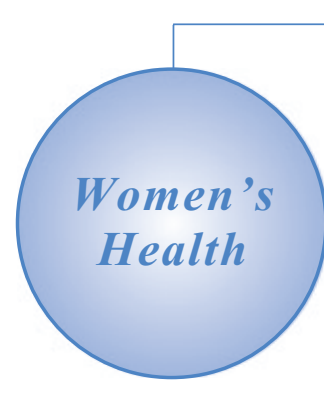

Heidi Collins Fantasia, PhD, RN, WHNP-BC, University of Massachusetts, Lowell, MA

Melissa Sutherland, $\mathrm{PhD}, \mathrm{RN}$, FNP-BC, Boston College, Chestnut Hill, MA

Holly B. Fontenot, RN, WHNP-BC, Boston College, Chestnut Hill, MA

Keywords

interpersonal violence contraception

Paper Presentation \section{6.}

\section{Methods}

Data were extracted from a sample of 2,000 medical charts. Inclusion criteria were female, reproductive age (menarche through menopause), and seeking reproductive health services. Institutional Review Board approval was granted for the study.

\section{Results}

Data analysis was performed using Statistical Package for the Social Sciences version 19. The overall rate of lifetime violence for this sample was approximately $28 \%$. The majority of the participants were between the ages of 15 and $26(79.1 \%)$. Regression analysis indicated that a history of lifetime violence was predictive of an increased number of contraceptive method changes in the previous year $(B=.283 ; p<.000)$. Additionally, a recent history of violence (past 12 months) predicted the use of a "hidden" method of contraception (injectables, implants, intrauterine devices, and sterilization) that is less vulnerable to partner interference or tampering $(B=.059 ; p$ $<$.000). A history of lifetime violence was also predictive of not using a contraceptive method since last menses $(B=.763 ; p<.000)$ and increased use of emergency contraception $(B=.796$; $p<$ .000).

Conclusion/Implications for Nursing Practice There is a high rate of intimate partner violence among young women seeking reproductive health services. Intimate partner violence affects patterns of contraception use and may place women who experience intimate partner violence at greater risk for unintended pregnancy due to increased method changes and lack of contraception. Nondaily contraceptive methods may be one alternative to increasing adequate contraception. Nurses who work with childbearing women need to assess for intimate partner violence when providing contraceptive counseling and discuss a wide range of contraceptive options that may increase the woman's control of use.

\title{
Self-Reported Explanations of Recurrent Chlamydia Infections and Urban Females
}

Melva Craft-Blacksheare, DNP, CNM, University of Michigan Flint, Detroit, MI

\section{Keywords} chlamydia reinfection urban teens

Women's Health Paper Presentation
Objective

$T$ o explore the essential elements of the lived experiences of urban adolescents diagnosed with recurrent chlamydia infection and to describe the meanings made of this phenomenon by the people experiencing it.

\section{Design}

A qualitative phenomenologic research study design was used, and open-ended interview questions were developed based upon the reviewed literature and the conceptual framework of the Health Belief Model.

\section{Setting}

Outpatient urban health clinics in Detroit, Michigan.

\section{Patients/Participants}

Ten urban Detroit adolescents, ages 15 to 19 , who self-reported recurrent chlamydia infections within the previous 12 months. 


\section{Methods}

Participants completed a demographic questionnaire and were interviewed using questions developed from the conceptual framework of the Health Belief Model. Sessions were audio taped. The tapes were transcribed and the transcripts reviewed to ensure accuracy. Hand-coding and a qualitative software package (Nvivo 8) were used to generate and classify codes. Themes were generated by the researcher who transcribed all interviews, read and reflected on transcripts, and reviewed field notes for overall meaning. To ensure the analysis process was systematic and verifiable, an expert with more than 25 years of experience in women's urban health was asked to review the data. Transcripts were discussed as a means to increase rigor and to discern any additional impressions of the data and ideas for additional themes and patterns.

\section{Results}

Participants expressed a heightened perceived susceptibility after recurrence of the disease. A knowledge deficit existed as of the seriousness of chlamydia, and its comparison to other sexually transmitted infections. The benefit of consistent condom use (to prevent future recurrence) was supported by nine participants. Many efficacious statements were expressed, suggesting a (new) self-empowerment position. Having a recurrent infection was a "wake up call" that encouraged participants to change and practice less risky sexual behaviors.

\section{Conclusion/Implications for Nursing Practice}

We investigated some of the underlying reasons urban adolescents continue to practice risky sexual behavior. The interviews offered an understanding of some psychosocial issues urban adolescents' experience upon which nurse practitioners can build better counseling and educational support. After obtaining this information, an evaluation of the existing procedures related to sexually transmitted infection follow-up can be compared, contrasted, and improved. Educating adolescents and offering support, which includes condoms, education, and counseling, are some of the necessary approaches suggested by participants in the study. The nursing community can develop new models of care to help eliminate this crisis based upon findings from this study.

\section{Families Planning Forward: Wholistically Processing a Rich Life}

\begin{abstract}
Objective
This his study is a follow-up to a qualitative study undertaken 15 years prior examining women's experiences in multiple role balancing. At the time of the original study, which was completed in 1994, women were entering the work force in record numbers. These women found themselves raising children in a different world than the one in which they grew up. At that time, the majority of women stayed home with the children, and the father was the breadwinner. Professional women were forced to make choices between conflicting demands of career and family and a prevalent theme in the literature focused on their feeling stressed and guilty. Societal change has evolved relative to the emerging employment trends of today's working mothers. In fact, the percentage of women in the workforce has actually been declining since entering the new millennium, a pattern that has not been demonstrated since the middle seventies.
\end{abstract}

\section{Design}

Grounded Theory based on Symbolic Interaction Theory.
Setting

Original and follow-up study participants were interviewed in either their homes, at work, or both.

\section{Patients/Participants}

A purposive sample of 17 women representing diverse professions, married and mothering at least one child under six were interviewed, and upon analysis a number of common themes developed, from which emerged three general categories: Wholistic Management, Support Resource Fit, and Balance as Process. Ultimately, the theory generated was entitled Women's Experiences Balancing Multiple Roles: Wholistically Processing On-Going Acceptable Peace. Seven of the original study participants were re-interviewed as to their continued experiences in balancing multiple roles.

\section{Methods}

These findings were compared to current research in multiple role balancing and to the findings from the original study.

Results

The aftermath study validated the thematic analysis and confirmed the findings of the original study. Additionally, due to emergence of
Susan Ann Murphy, PhD, APRN, WHNP, Rivier College, Nashua, $\mathrm{NH}$

Keywords

balancing woman

working health

Women's Health Poster Presentation 
new data from a retrospective view, the theme regarding spousal involvement was renamed, now Spouse as Partner, and reconfigured, and two new themes emerged: Using Diverse Resources and Import/Need to Work. In accordance, the original categories have been adjusted, and the evolving theory has been revised based on a developmental perspective, and is now titled Families
Planning Forward: Wholistically Processing a Rich Life.

Conclusion/Implications for Nursing Practice

Knowing that stress can lead to the development of adverse health concerns, an enlightened understanding of the actual experiences is essential in providing health care to this patient population.
Janelle Gardner, RN, MSN, $\mathrm{PhD}$, California State University, Chico, Chico, CA

\section{Keywords}

HIV

AIDS

Kenya

phenomenology

\section{Women's Health} Poster Presentation

\section{Understanding the Lived Experience of HIV Positive Women in Kenya: A Global Perspective}

Objective

K enya has approximately 1.4 million adults infected with HIV/AIDS, with a national prevalence rate of approximately $7.4 \%$. The majority of the Kenyan people have not participated in a voluntary counseling and testing program and do not know their HIV status. This increases the likelihood of infecting others and spreading the disease. The purpose of this phenomenologic study was to explore HIV positive women's experiences and perceptions about voluntary counseling and testing programs.

\section{Design}

The literature suggests that there is a lack of qualitative research in Africa related to voluntary counseling and testing. A qualitative phenomenologic study was conducted.

\section{Setting}

All of the participants included in the study lived in a rural village in western Kenya. The investigator and translator walked to the participants' homes in the poor rural village on dirt paths. The interviews were conducted inside the women's homes.

Patients/Participants

A total of 29 women participated in the study. All of the women were HIV positive. The participants ranged from 22 to 63 years of age and were members of two tribes, the Luo and Luyhia.

\section{Methods}

A semistructured interview guide with open-ended questions followed by probe questions was used to elicit in-depth responses during the interviews. The length of the interviews varied between 45 and 60 minutes. All the interviews were audio taped and transcribed verbatim. Moustakas' framework for qualitative analysis was the primary model for analyzing data in this study.

\section{Results}

Six themes emerged while analyzing the data: living in fear, making the decision to be tested, the journey toward acceptance, changing behavior, planning for the future, and encouraging others to be tested. The themes were common to all or almost all of the participants.

\section{Conclusion/Implications for Nursing Practice} HIV/AIDS continues to be a major public health issue in Kenya and other countries in sub-Saharan Africa. Only $36 \%$ of the Kenyan adults have participated in voluntary counseling and testing and know their HIV status. Voluntary counseling and testing programs are crucial in attaining goals related to health promotion, prevention, and management of the disease. Issues concerning the acceptance and use of voluntary counseling and testing provide valuable information for enhancing access and the quality of the program. This study expands our understanding of the lived experience of HIV positive women and their perceptions of voluntary counseling and testing programs. 


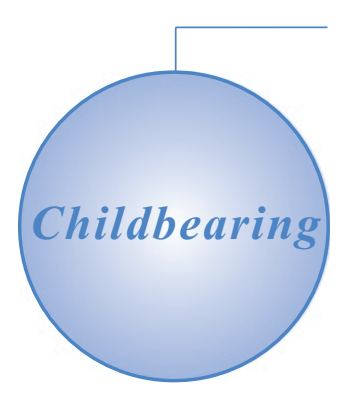

Paul D. Loprinzi, PhD, Bellarmine University, Louisville, KY

Bradley J. Cardinal, PhD, Oregon State University, Corvallis, OR

Elizabeth M. Fitzgerald, EdD, APRN, PMHCNS-BC, LMFT, Bellarmine University, Louisville, KY

Keywords exercise mental health pregnancy psychology women

\section{Paper Presentation}

\section{Association Between Objectively Measured Physical Activity and Depression Among a Nationally Representative Sample of U.S. Pregnant Women}

Objective

$\mathrm{D}$ epression affects approximately $20 \%$ of pregnant women. Pregnant women who experience depression are at greater risk for postpartum depression and their offspring's health may be compromised. Psychological and pharmaceutical therapy has been shown to reduce rates of depression; however, such treatments can be costly, time-intensive, and few medications have been established as unquestionably safe during pregnancy. Additional strategies that complement these therapies are needed, which may include regular engagement in physical activity. Empirical evidence indicates that physical activity is associated with reduced depression symptoms among pregnant women. However, all studies have used self-report measures, which are subject to item interpretation, recall bias, and social desirability effects. To provide an accurate understanding of the relationship between physical activity and depression among pregnant women, we examined this association while employing an objective measure of physical activity (i.e., accelerometry) among pregnant women.

\section{Design}

A cross-sectional study design using a representative sample of noninstitutionalized U.S. civilians, selected by a complex, multistage probability design.

Setting

Data from the 2005-2006 National Health and Examination Survey were used.

\section{Patients/Participants}

One hundred and forty-one pregnant women (determined from a urine and serum blood sample) were part of the study.

Methods

These participants completed the Patient Health Questionnaire-9 to assess depression status and provided at least 4 days of $10+$ hours of monitoring data (ActiGraph 7164 accelerometer).

Results

More than $19 \%$ of the pregnant women experienced some depression and compared to their nondepressed counterparts, they were less physically active. Pregnant women who were not depressed spent significantly $(p<.05)$ more time in moderate-to-vigorous physical activity than pregnant women with some depression $(M=14.49 \pm$ 1.29 minutes/day vs. $M=8.75 \pm 1.98$ minutes/day, respectively). With regard to meeting physical activity guidelines (i.e., 150 minutes of moderate-tovigorous physical activity or 75 minutes of vigorous physical activity per week), a smaller percentage of depressed pregnant women $(4.79 \% \pm 1.47)$ met guidelines compared to nondepressed pregnant women $(20.89 \% \pm 7.99 \%$; $p<.01)$. Controlling for age, race-ethnicity, marital status, smoking status, body mass index, and gestation, the odds ratio for meeting physical activity guidelines was 10.73 (95\% Cl: 0.88-130.68; $p=.06$ ) for nondepressed pregnant women, compared to depressed pregnant women.

Conclusion/Implications for Nursing Practice When feasible, obstetricians and nurses should encourage pregnant women to engage in safe forms of physical activity on a regular basis.

\section{Babies and Mothers: Skin-to-Skin Immediately After Birth}

Holly Champagne, RNC, MSN, Objective

CNS, Kaiser Permanente,

Roseville, CA
$T$ o have $100 \%$ of medically stable mothers and infants spend greater than 30 minutes skin-toskin within the first hour of birth.

Design

Quality improvement implementation project.

\section{Patients/Participants}

Mother/infant dyads immediately after birth, labor and delivery nurses, and respiratory therapists present in the first hour after delivery.

Methods

The quality improvement method used at this hospital was MAP-IT. Mobilize: An interdisciplinary 
Keywords

skin-to-skin

bonding

breastfeeding

quality improvement

implementation

\section{Childbearing \\ Paper Presentation}

project leadership team was formed and sustained. The team included the birth center nurse manager, educator, neonatal resuscitation team respiratory therapist, a lactation consultant, and a registered nurse project leader. Assess: Skinto-skin frequency and registered nurse practices and knowledge were assessed pre-, during, and postimplementation. Frequency of timed skin-toskin contact was recorded in patients' electronic health records. Plan: The team developed a plan with short- and long-term objectives summarized using a logic model. Implementation: Nursing and respiratory staff members were educated about benefits of mother/infant skin-to-skin contact, strategies to implement the practice, and the process for accurate documentation. Track:
Monthly data were evaluated and project adjustments made. Graphs provided visual information to staff and leadership about the steady improvement in accurate documentation and rates of skinto- skin contact.

\section{Results}

Accurate skin-to-skin documentation increased from $40 \%$ to $90 \%$ over a 6 -month period. Skinto-skin contact for 30 minutes or more increased from $58 \%$ to $83 \%$.

Conclusion/Implications for Nursing Practice A multidisciplinary team with a comprehensive plan and sustained focus led to more frequent skin-to-skin contact.

\section{A Renewed Commitment to Improving Quality and Efficiency of Postpartum Education During Hospitalization}

Barbara L. Buchko, DNP, RN, York Hospital, York, PA

Connie H. Gutshall, MS, RN, NE-BC, York Hospital, York, PA

\section{Keywords} postpartum period postnatal care teaching patient education

\section{Childbearing Paper Presentation}

\section{Objective}

$D$ rior to discharge from the hospital, the postpartum mother is expected to demonstrate knowledge and confidence in her ability to provide adequate care for herself and her newborn. Mother-baby nurses at our hospital expressed concerns for meeting educational needs of new mothers citing limited time and too much information, whereas patient satisfaction scores demonstrated poor results for consistency of information. A renewed commitment was made to improve the quality and efficiency of postpartum education. The Johns Hopkins Nursing Evidence-Based Practice Model was used to evaluate the literature. Recommendations from the evidence suggested collaborating with the new mother to create an individualized education plan and reinforcing verbal instruction with written information. The purpose of this project was to investigate whether the implementation of an evidence-based streamlined education process (comprehensive education booklet, individualized education plan, and integration of education into the clinical pathway) and nurse education would improve the quality and efficiency of postpartum education during hospitalization.

\section{Design}

A pre- and post test design was used to measure quality of discharge teaching for new mothers and efficiency of the teaching process for registered nurses before and after implementation of the intervention.

Patients/Participants

A convenience sample of 100 new mothers (50 receiving usual care and 50 receiving interven- tion) completed the "Quality of Discharge Teaching Scale" before leaving the hospital. Thirty-one registered nurses caring for mother-baby dyads completed the "Questionnaire on Factors Influencing Patient Teaching" before and after implementation.

\section{Methods}

Independent samples $t$ test was used to examine differences in mothers' perceived need for education, amount of education received, and quality of teaching. Paired samples $t$ tests were used to assess nurses' perception of efficiency of patient teaching. A team of mother-baby nurses created a comprehensive education booklet to promote consistent information, documentation to create an individualized education plan, integration of education into the clinical pathway, and mandatory education about the process.

\section{Results}

A difference in the quality of education was not found, yet mothers in both groups reported receiving adequate information and good-quality teaching. A significant increase from pre- to postintervention $(p<.01)$ was found for availability of patient teaching materials when providing patient teaching. Patient teaching forms provided added guidance to nurses for teaching and documentation $(p<.01)$.

\section{Conclusion/Implications for Nursing Practice}

This educational intervention shows how a comprehensive educational booklet and enhanced documentation can improve efficiency in the patient teaching process for nurses. 


\section{Higher Carbohydrate Versus Higher Fat Diet in Gestational Diabetes: A Pilot Study}

\begin{abstract}
Objective
T he historic practice of advising a lowcarbohydrate and higher fat diet in the management of gestational diabetes mellitus has not been sufficiently tested. In addition to glucose, high maternal triglycerides and free fatty acids are independent risk factors for fetal macrosomia and excess neonatal adiposity. We tested the hypothesis that a higher complex carbohydrate/lower fat diet would result in higher postprandial glucose excursions but an overall 24-hour glucose areaunder-the-curve resulted that is no different or lower than that of a low-carbohydrate/higher fat diet.
\end{abstract}

\section{Design}

Randomized crossover study.

Setting

Clinical Translational Research Center.

Patients/Participants

Women with diet-controlled gestational diabetes mellitus.

\section{Methods}

Women with diet-controlled gestational diabetes mellitus consumed a high complex carbohydrate/ low-fat diet (HC/LF; $60 \% \mathrm{CHO}, 25 \%$ fat, and $15 \%$ protein) and a low carbohydrate/higher fat diet (LC/HF; 40\% carbohydrate [CHO], 45\% fat, and $15 \%$ protein) for 3 days each (washout in between) while wearing a continuous glucose monitor. On day four of each diet, postprandial lipemia was measured hourly for 5 hours after women consumed breakfast ( $30 \%$ of total daily calories). All food was provided by the Clinical Translational Research Center. A paired $t$ test was used for difference testing.
Results

Ten women with gestational diabetes mellitus (Mean \pm SEM; body mass index $33.6 \pm 1.5 \mathrm{~kg} / \mathrm{m}^{2}$; age $29 \pm 1$ years; $30.4 \pm 0.5$ weeks gestation) completed the diet crossover. Whereas continuous glucose monitor revealed no difference in mean nocturnal blood glucose (BG) or fasting $B G$ $(p>.05)$, the $\mathrm{HC} / \mathrm{LF}$ diet yielded a modestly higher level: mean 24 -hour BG $(96.3 \pm 3.4$ vs. $90.3 \pm 3.8$ $\mathrm{mg} / \mathrm{dl}, p=.03$, respectively), mean 24-hour areaunder-the-curve, and mean postprandial 2-hour area-under-the-curve across meals $(p<.005)$. One- and two-hour postprandial glucose by continuous glucose monitor were higher across meals on the HC/LF diet compared to the LC/HF diet (115 \pm 3 vs. $106 \pm 3 \mathrm{mg} / \mathrm{dl}[p=.009]$ and $108 \pm 5$ vs. $98 \pm 3 \mathrm{mg} / \mathrm{dl}$ [ $p=.01]$, respectively). On day four, there were no differences in fasting plasma glucose, insulin, triglycerides, or free fatty acids. Postprandial blood analyses revealed higher 5hour area-under-the-curve for glucose and insulin on the HC/LF diet ( $p=.004$ for both), no difference in the 5-hour triglycerides area-under-the-curve, but a higher 5 -hour free fatty acids area-underthe-curve on the HF/LC diet $(p=.005)$.

Conclusion/Implications for Nursing Practice

The pattern of glycemia on both diets was remarkably similar. Despite modestly higher glucose concentrations on the HC/LF diet, both diets easily met current recommendations ( $<140$ and $<120$ $\mathrm{mg} / \mathrm{dl}$ at 1 and 2 hours postprandial; mean BG 87-104 mg/dl). Thus, nurses may be able to help women with gestational diabetes mellitus consume a more balanced diet (in carbohydrate/fat) while still meeting glycemic targets. The consistently higher free fatty acids on the LC/HF diet could worsen maternal insulin resistance and augment neonatal adiposity. Further investigation is required to understand the importance of balancing dietary carbohydrate and fat by its metabolic effects on mother and offspring.
Teri L. Hernandez, PhD, RN, University of Colorado Denver, Aurora, $\mathrm{CO}$

Linda A. Barbour, MD, MSPH, University of Colorado Denver, Aurora, CO

Jacob (Jed) E. Friedman, PhD, University of Colorado Denver, Aurora, CO

Melanie S. Reece, $\mathrm{PhD}$, University of Colorado Denver, Aurora, CO

Molly A. Krause, MS, RD, University of Colorado Denver, Aurora, CO

Rachael E. Van Pelt, PhD,

University of Colorado Denver, Aurora, CO

Keywords GDM

diet

carbohydrate

Childbearing

Paper Presentation

\section{Women's Self-Competence for Childbirth}

\section{Objective}

C hildbirth in America is changing. With today's escalating elective induction of labor and cesarean rates and their potential negative health outcomes, it is essential to identify women who embody effectiveness, ability, sufficiency, and/or success in childbirth; in other words, women who are self-competent. The purposes Tanya Tanner, RN, CNM, MS, of this study were to investigate expert mater- MBA, Frontier Nursing nity care providers' descriptions of, understand- University, Northglenn, $\mathrm{CO}$ ing of, and clinical experiences with women who Nancy K. Lowe, CNM, PhD, exhibit self-competence for childbirth and to iden- FACNM, FAAN, University of tify the defining attributes of self-competence for Colorado Denver, Aurora, CO childbirth. 
Keywords

self-competence for childbirth

Delphi method

expert maternity care provider

\section{Childbearing}

Paper Presentation
Design

A Delphi study consisting of an expert panel of 108 maternity care providers was undertaken. Four survey rounds designed to first elicit qualitative opinions and subsequently bring the panel to consensus about the characteristics, antecedents, and outcomes of women who are self-competent for childbirth were conducted.

\section{Setting}

A variety of birth settings from across the United States.

\section{Patients/Participants}

One hundred and eight panelists (13 doulas, 19 nurses, 48 midwives attending births in homes, birth centers and hospitals, and 28 family practice physicians, obstetricians/gynecologists, and perinatologists), certified, licensed, or registered in their area of expertise who have provided handson care to laboring women for at least the past 5 years.

Methods

The first qualitative Delphi study round identified panelists' opinions regarding the antecedents, characteristics, and outcomes associated with women who are self-competent for childbirth.
Coded statements from this round formed the basis of the second and third rounds, which elicited group consensus. The final (fourth) survey round was a "member check"; panelists declared their degree of agreement with the final study results and identified whether results described their nulliparous patients.

\section{Results}

First round qualitative content analysis resulted in more than 400 codes that were condensed into 195 statements ranked in subsequent Delphi rounds. In the second and third round analysis, we identified 62 consensus statements. Fourth round analysis resulted in continued panel agreement

Conclusion/Implications for Nursing Practice

Study results provide increased understanding of the phenomenon of self-competence for childbirth and provide conceptual validation and an item pool for subsequent instrument development measuring self-competence for childbirth. Results also provide a basis for discussion among maternity care providers about how patients cope with their individual labor and delivery experiences, and may provide the basis for a more standard nomenclature identifying women who are selfcompetent in childbirth.

\section{Physical Activity and Diet During Pregnancy: What Low-Income, Pregnant African American Women Think}

Susan W. Groth, PhD, RN, WHNP-BC, University of Rochester, Rochester, NY

\section{Keywords}

pregnancy

diet

physical activity

Childbearing
Paper Presentation

Objective

T

o gain insight into how low-income, pregnant

African American women viewed physical activity and how they approached nutrition during pregnancy.

Design

Descriptive study using three focus groups.

\section{Setting}

Women were recruited from urban prenatal care sites and the Special Supplemental Nutrition Program for Women, Infants and Children (WIC) services in a medium-sized urban northeastern city.

Patients/Participants

Twenty-six adult, low-income, pregnant African American women, aged 18 to 39 years old, the majority of which were within the first 20 weeks of pregnancy.

Methods

Three focus groups were conducted utilizing open-ended questions related to physical activity and dietary practices during pregnancy. Content analysis was used to analyze the verbatim tran- scripts. Analysis focused on meaning, intention, and context. Groups were compared and contrasted at the within- and between-group levels to identify themes.

Results

Two themes were identified that provided insight into how women viewed physical activity during pregnancy: (a) fatigue and low energy dictate activity, and (b) motivation to exercise is not there. Three themes were identified that related to diet: (a) despite best intentions appetite, taste, and cravings drive eating behavior, (b) l'll decide for myself what to eat, and (c) eating out is a way of life.

Conclusion/Implications for Nursing Practice Women reported that being physically active and improving their diets was not easy. Most women indicated they had decreased their physical activity since becoming pregnant and those inactive before pregnancy did not plan to become active. Attempts at improving diets were undermined by frequenting fast food restaurants and cravings for highly dense, palatable foods. Women ceded to the physical aspects of pregnancy, often 
choosing to ignore the advice of others. A combination of low levels of physical activity and calorie-dense diets increased the risk of excessive gestational weight gain in this sample of women, consequently increasing the risk for weight retention after pregnancy. Nurses need to be creative when promoting physical activity and healthy diets during pregnancy. Building on the idea of listening to their bodies, nurses can encourage women to listen to other aspects of their bodies by encouraging healthy foods and activities women enjoy on good days. Nurses can query women about beliefs regarding physical activity and diet and offer information to ensure understanding of what contributes to healthy pregnancy outcomes. Intervention can focus on factors such as cravings and what tastes good, suggesting ways to manage these pregnancy effects within a healthy diet.

\section{Fall Risk Screen for the Postepidural, Postpartum Patient}

Objective

$D$ ostpartum patients who receive an epidural

infusion during labor for pain control are at increased risk of falling when attempting to ambulate for the first time. A comprehensive literature review revealed only one obstetric fall risk screen that had not been tested in the clinical setting. No research was found that addressed the unique situation of the postepidural, postpartum patient. The objective of this study was to determine whether return of motor function in the lower extremities could be used as the marker for safe ambulation.

\section{Design}

Descriptive, observational design.

\section{Setting}

Low/moderate risk intrapartum/postpartum patients who received an epidural for pain control during labor.

\section{Patients/Participants}

One hundred low/moderate risk obstetric patients with an epidural. Exclusion criteria included cesarean birth and Baseline Muscle Strength Scale of $<4$.

\section{Methods}

Obstetric registered nurses assessed study patients' deep tendon reflexes and motor strength using the Motor Strength Scale prior to receiving Shirley Warren, RN, MS, epidural anesthesia and again prior to ambulating WHNP-BC, University of postpartum. If a subject's deep tendon reflexes Rochester Medical Center, and/or Motor Strength Scale scores did not return Rochester, NY to baseline following receipt of epidural anesthe- Keywords sia, the subject was not ambulated and was re- postpartum assessed prior to next ambulation attempt. If a epidural subject's scores returned to baseline, the subject was assisted with ambulation. The nurses evaluated the subject's success in ambulation using the following criteria: ambulating without lower extremity weakness or buckling of knees. Data were gathered and entered by the registered nurses onto the data sheet assigned to each subject.

\section{Results}

Data were collected on 100 subjects. Of this number, 91 (91\%) subjects successfully ambulated. Nine $(9 \%)$ required assistance on ambulation. There were no falls.

\section{Conclusion/Implications for Nursing Practice}

The results supported the assessment of return to baseline levels deep tendon reflexes and Motor Strength Scale for indication of readiness to ambulate. The results of this study support the addition of these assessments into the standard of care for the postpartum patient.

\section{Childbearing}

Poster Presentation

\section{Having an Elective Cesarean: Doing What's Best for Me}

\section{Objective}

To o determine how women decide to deliver their infants by cesarean instead of experiencing a trial of labor and expected vaginal delivery when appropriate. The specific goals were to answer the research questions: What is the decision-making process by which healthy, low risk women choose to give birth to their infants by cesarean in the absence of medical indications? What antecedents occur to influence a pregnant woman's decision to undergo a maternal request Cynthia Acuff Michaluk, RN, cesarean? $\mathrm{PhD}$, Pediatrix Medical Group, Knoxville, TN

Design

Exploratory study. Symbolic interactionism and Keywords feminism were utilized to provide a theoretical cesarean framework for the study. The grounded theory childbirth methodology by Strauss and Corbin was used to develop the core category, context, antecedents, intervening factors, and consequences. 
Setting

Knox and Sevier Counties in East Tennessee.

Patients/Participants

Seven women underwent in-depth interviews. To qualify for the study, the women had to be healthy and low-risk, had an elective cesarean birth within the last 2 years, be 18 years or older, and reside in East Tennessee.

Methods

Grounded Theory Methodology by Strauss and Corbin.

Results

From the data, a substantive theory was identified, "Having an elective c-section: Doing what's best." Mothers voiced that they felt like having a cesarean was best for them and their infants. The antecedents of the women's decision were being scared and perceiving a cesarean as an easier way to give birth. Women made this choice after gathering information and seeking support from healthcare providers, friends, and family within the context of progressing through the pregnancy. Intervening facilitating factors included receiving support from others and the ability to schedule the cesarean. Hindering factors were nonsupportive people and inappropriate medical information. Once the decision was made and the cesarean was performed, the women voiced happiness with their decision.

Conclusion/Implications for Nursing Practice

The findings of this study may assist office nurses, public health nurses, midwives and advanced practice nurses, childbirth educators, and other women's health nurses to educate women that childbirth is a normal process and vaginal birth can be achieved in most women safely. These providers can educate women on the risks/benefits of both birthing options. Recommendations are given for further research.

\section{Correlates of Perinatal Depression in Diverse, Low-Income Women}

Deborah Pollard, PhD, RNC, CNE, IBCLC, University of North Carolina Wilmington, Wilmington, $\mathrm{NC}$

Judith Jarosinski, PhD, RN, CNE, Salisbury University, Salisbury, MD

Keywords postpartum depression self-efficacy social support

\section{Childbearing} Poster Presentation
Objective

To examine the prevalence of perinatal depression and the risk factors and related variables affecting the occurrence of depression in a group of diverse, low-income women. The study contrasted the relationship between sociodemographic variables, depression, self-esteem, self-efficacy, maternal confidence, social support, and stress.

Design

A descriptive, correlational design incorporating qualitative and quantitative research methods.

\section{Setting}

A health department in Southeast North Carolina.

Patients/Participants

A convenience sample of 60 , low-income mothers participating in the prenatal program at the health department were surveyed during the third trimester of their pregnancies and again between 6 and 8 weeks following birth.

\section{Methods}

Focus groups were used to explore perceptions and experiences during the perinatal period. In addition, participants completed a demographic questionnaire, Beck Depression Inventory, Edinburgh Postnatal Depression Scale, Rosenberg Self-Esteem Scale, General Self-Efficacy Scale, Stress Scale, Maternal Confidence Questionnaire, and the Multidimensional Scale of Perceived Social Support.

\section{Results}

The mean age of the participants was 25.4 years, $50.8 \%$ had completed some college/college degree, and $26.2 \%$ reported past history of depression. Of the sample, the majority were White (52.5\%), 27.9\% Black, and 19.7\% Hispanic. A majority of the participants (60.7\%) scored above 10 on the Beck Depression Inventory during the third trimester whereas $44.2 \%$ scored above 10 on the Beck Depression Inventory at 6 to 8 weeks postpartum. Moderate depression was reported by $24.6 \%$ of the participants during the third trimester of pregnancy and $13.4 \%$ reported moderate depression at 6 to 8 weeks postpartum. There were significant positive correlations between the Beck Depression Inventory and Edinburgh Postnatal Depression Scale at both time periods $(r=.582$, $p=.000$ and $r=0.738, p=.000$ ). There were no significant differences in mean depression scores across ethnic groups for either time period ( $F=$ 0.997, $p=$.375; $F=0.051, p=.950)$. There were significant negative correlations between depression level, self-esteem, and social support and age and general self-efficacy. There were significant positive correlations between self-esteem, social support, and self-efficacy. 
Conclusion/Implications for Nursing Practice Study results demonstrated that there were no significant differences in depression levels across ethnic groups, however participants overall reported significantly higher depression levels in the third trimester of pregnancy as compared with 6 to 8 weeks postpartum. These findings support the need for heightened assessment and management of depression not only after delivery, but also during pregnancy. In addition, self-reports of mood changes before pregnancy were significantly related to depression scores during the third trimester of pregnancy and at 6 to 8 weeks postpartum.

\section{Disaster and Diaspora: Mental Health Status of Childbearing Women Living Through Disaster Recovery}

\begin{abstract}
Objective
T he Hurricane Katrina disaster provides a model to study pregnant women affected by a chronic stressful life course. Over the past 6 years, women in this study lived through a catastrophic disaster, family and home disruption, relocation to temporary communities, and move back to New Orleans to live. Women who became pregnant after the return to New Orleans also negotiated the stresses of living in the recovering communities with reduced access to health care and neighborhood instability. Immigrant women who moved into the city during the recovery period also faced a more stressful environment. Data are needed to describe the long-term effects of disaster recovery experiences on maternal stress and mental health risks.
\end{abstract}

Design

Cross-sectional, descriptive study.

Setting

Recruitment and data collection occurred at community prenatal care clinics, Healthy Start, and hospital prenatal classes in the greater New Orleans area.

Patients/Participants

Prenatal women ( $n=222,24-40$ weeks gestation), currently enrolled in prenatal care, and living in the greater New Orleans area.

\section{Methods}

Measures analyzed included previous disaster exposure, perception of disaster recovery, depression (Edinburgh Depression Scale [EDS]), posttraumatic stress disorder (Post-traumatic Check- list), and pregnancy-related distress (Lobel scale). Gloria P. Giarratano, APRN, Linear regression was used to model mental health CNS, PhD, Louisiana State outcomes, with adjustment for race, marital status, University Health Sciences education, employment, age, and smoking.

Results

Women were predominately African American $(70 \%)$, single $(72 \%)$, and with income $<\$ 15,000 /$ year $(58 \%)$. Disaster exposure was moderately high: $29 \%$ walked through flood waters; $61 \%$ had some or enormous damage to their homes; and 10\% saw someone die. Mental health measures indicated $56 \%$ scored "at risk" for depression (EDS > 8), whereas 11\% were likely experiencing PTSD. Women who reported high exposure to Katrina scored significantly higher on the post-traumatic stress disorder (PTSD) and pregnancy-related distress scales, and the feeling that one's life was still disrupted (36\%) was strongly associated with depression and pregnancy-related distress $(p<.01)$, even after adjustment for experience of the hurricane. Lifestyle practices such as smoking (14\%) were associated with depression and higher perceived stress $(p<.01)$. Higher social support, optimism about the future, and use of massage were associated with better mental health.

Center, New Orleans, LA

Jane Savage, RN, PhD, LCCE, Loyola University, New Orleans, LA

Susan Rick, RN, DNS, Louisiana State University Health Sciences Center, New Orleans, LA

Emily Harville, PhD, Tulane University, New Orleans, LA Veronica Barcelona de Mendoza, MSN, MPH, RN, APHN-BC, Louisiana State University Health Sciences Center, New Orleans, LA

Keywords

disaster

PTSD

stress

perinatal depression

\section{Childbearing}

Poster Presentation

Conclusion/Implications for Nursing Practice

Past exposure to hurricane disaster and current recovery status contribute to mental health problems. Pregnant women with a history of disaster exposure need ongoing mental health assessment and prenatal care that address this risk. Lifestyle choices such as smoking require further intervention to prevent perinatal risks. 


\section{A Comparison of Traditional Paper, Computer Screen, and Computer Printout Interpretation of Electronic Fetal Monitoring Tracings}

Mary T. Zabielski, RN, PhD, Magee-Women's Hospital of UPMC, Pittsburgh, PA

Keywords

electronic fetal monitoring EFM tracing interpretation EFM research

\section{Childbearing \\ Poster Presentation}

Objective

L ospitals are implementing paperless systems for electronic fetal monitoring interpretation. With the introduction of these systems, electronic fetal monitoring tracings are visually interpreted using computer screens and computer printouts instead of the traditional, scrolling paper mode. The history of electronic fetal monitoring and established interpretation theory has been based on the visual interpretation of paper tracings. The process of interpretation using computer modes is potentially different. Each mode allows a different amount of tracing to be viewed simultaneously, produces an image in different colors and with different reference lines, and involves either static or moving images. A concern is that all three modes are used interchangeably without proof that a satisfactory correlation exists. Is there a difference in electronic fetal monitoring tracing interpretation depending on the mode used?

\section{Design}

Retrospective, descriptive correlational study of electronic fetal monitoring tracing records.

Setting

The birth center of a large, university medical center.

\section{Patients/Participants}

The tracing records of 13 patients were collected and reviewed by five experienced intrapartum registered nurses.

Methods

For each patient in each mode, $1 \frac{1 / 2}{2}$ to 2 hours of tracings were collected. Tracings were random- ized and then assessed for uterine activity and fetal heart rate characteristics and assigned an interpretative label every 15 minutes (6-8 assessments per tracing, $N=1,515$ ).

\section{Results}

Cross tabulation using chi-square analysis was performed. A significant difference $(p=.26)$ in perception of decelerations was found. More decelerations were identified in the computer screen mode. In addition, there was a significant difference $(p=.009)$ in the interpretative label assigned to the tracing. More tracings were assessed as nonreassuring in the computer screen mode.

\section{Conclusion/Implications for Nursing Practice}

The visual interpretation of fetal heart rate (FHR) tracings is used to assess fetal well-being, the presence of labor, and the adequacy of labor. The accuracy of this interpretation fundamentally affects the care of the pregnant woman and fetus. Most hospitals use some combination of computer-generated FHR images and paper tracing images for bedside interpretation, central surveillance, "down times," and archiving. The assumption that these modes are interchangeable may be suspect given the findings of this study. Poor correlation between visual modes potentially could lead to inaccurate assessments, inconsistent communication, inappropriate interventions, and increased vulnerability during litigation. More research is needed to either support or refute the equivalence of the three modes when interpreting electronic fetal monitoring tracings.

\section{Unexpected Effects of Reducing Elective Inductions at Less Than 39 Weeks Gestation}

Vicki A. Lucas, RNC, BSN, MNEd, WHNP, PhD, PeriGen, Inc. and Vicki Lucas, LLC, Phoenix, MD

\footnotetext{
Objective

Study the clinical outcomes of an intervention $\bigcirc$ to comply with the Institute for Healthcare Improvement's 39-week induction initiative.
}

\section{Setting}

Hospital settings ranging from small rural community to large urban teaching hospitals.
Design

Retrospective study.
Patients/Participants An aggregate of 157,283 births between 2007 and 2011. 


\section{Methods}

All hospitals were participating in a quality improvement program with two common aspects. First, an intelligent medical record (PeriBirth, Princeton, $\mathrm{NJ}$ ) that recognizes the intention to deliver or induce electively at less than 39 weeks gestation and requests written justification. Second, structured quarterly performance reviews using While to mention PeriBirth once, as the tool used in the study; PeriBirth is owned by PeriGen, and additional mentions becomes promotion. Intelligent medical record reports on rates of elective induction or cesarean at less than 39 weeks gestation and other related topics. In addition, some participating institutions had formal objectives to reduce the rate of elective births at less than 39 weeks gestation, others did not. Electronic birth records were examined for elective inductions at less than 39 weeks gestation, all inductions at less than 39 weeks gestation, primary cesareans, and 5-minute Apgar scores.

Results

Rates fell for elective induction at less than 39 weeks gestation* $0.98 \%$ to $0.53 \%$, induction at less than 39 weeks for any reason* $11.7 \%$ to $9.5 \%$, and most clinically significant, for all births at less than 39 weeks* $43.9 \%$ to $38.0 \%$. The rates of primary cesarean and 5-minute Apgar scores between 0 and 6 showed no change during the study period. Overall induction rates* rose during the study period from $32.6 \%$ to $35.9 \%$. $\left({ }^{*} p<.0001\right.$ for trend).

Conclusion/Implications for Nursing Practice

The intervention was highly successful in reducing elective inductions at less than 39 weeks gestation without an increase in the rate of primary cesarean or low 5-minute Apgar scores. In addition, clinical behavior changed beyond the strict confines of "elective" induction, as we observed an unexpected decrease in induction rates at less than 39 weeks for any indication. This translated to a large reduction in the rate of birth at less than 39 weeks. Furthermore, these hospitals have greatly exceeded a recommended benchmark of $<5 \%$ for elective birth at less than 39 weeks gestation with their aggregate rate of $1.4 \%$ in 2011 . With focused attention, communication, and technologic tools, providers will change their clinical behavior, which may have positive clinical outcomes on the patients. Nursing strategies to maintain the gain will involve further research and techniques to engage the providers.
Keywords

reduction in labor inductions

clinical outcomes

Childbearing

Poster Presentation

\section{A Fresh Look at the Postpartum Period: New Mother's Needs During the First Months at Home}

\section{Objective}

$\mathrm{n}$ an effort to provide new mothers with appropriate interventions and services during the transitional time frame of the postpartum period, every effort must be made to ensure that services provided are tailored to the needs of these women. Needs of the current generation of American women becoming mothers were unexplored. The purpose of this study was to discern the perceived needs of a sample of American women during the postpartum period following hospital discharge and to relate their needs to postpartum health care.

\section{Design}

A qualitative descriptive research study was conducted.

\section{Setting}

Twenty-one face-to-face interviews were conducted primarily at the women's homes, their places of employment, or a coffee shop at the women's discretion. Three telephone interviews occurred with women outside of southeastern Pennsylvania.

\section{Patients/Participants}

Twenty-four low-risk postpartum women from 6 weeks to 13 months postpartum were identified from a criterion-based snowball sampling of the community, primarily from Southeastern Pennsylvania

\section{Methods}

Digitally recorded semistructured interviews of approximately 60 minutes in length were conducted. Data were transcribed verbatim and content analyzed. Multiple strategies were used to ensure trustworthiness of the findings, including member checks, bracketing, and an audit trail.

\section{Results}

Seven themes were identified: upheaval, seeking a new social network, expanding the new mother's definition of self, is it possible to prepare someone to become a mother, breastfeeding - the need for support, what to do with a baby-am I doing it right, and postpartum services redesigned. Results were shared with participants.
Suzanne F. Foley, WHNP-BC,

PhD, RNC, Widener

University, Chester, PA

Keywords

postpartum follow-up community-based postpartum

care

postpartum needs post-hospital discharge postnatal care

becoming a mother

\section{Childbearing}

Poster Presentation 
Conclusion/Implications for Nursing Practice

The need for professional support through community-based interventions after hospital discharge was the overarching need identified. Professional postpartum follow-up was found lacking, primarily related to women's postpartum mental health and breastfeeding support needs. Multiple implications for nursing practice, education, and research are discussed.

\section{Preventing Childbearing-Related Obesity: Women's Perceived Needs}

Kristen S. Montgomery, PhD,

$\mathrm{RN}$, University of North

Carolina at Charlotte,

Charlotte, $\mathrm{NC}$

Lindsay F. Schalles, BSN, RN, University of North Carolina at

Charlotte, Charlotte, NC

Keywords

pregnancy weight gain

postpartum weight loss

\section{Childbearing}

Poster Presentation

\section{Objective}

To determine what women perceive they need to avoid excessive weight gain related to childbearing.

\section{Design}

Descriptive qualitative interviews.

\section{Setting}

Interviews at a place that was convenient to the women.

\section{Patients/Participants}

Six pregnant women and seven women with chil-

dren 7 years or older participated. All were 18 or older. These criteria were chosen to capture the pregnancy perspective and the longer term perspective. The 7-year time frame was chosen so women would have ample time to lose postpartum weight and so they could reflect back on what might have been helpful. Also, this time frame would put them beyond the initial all-consuming childcare demands of the newborn period. This is also the period of time when children start to attend a full day of school, which would allow more women the possibility of participating and the mental resources to devote to the task.

\section{Methods}

Interviews began with the lead question: "Please tell me what you need (needed) to prevent longterm weight gain from your pregnancy." Probes were used as needed to solicit additional details. Women were also encouraged to focus on the broad perspective of what they might need, including governmental policies and environmental changes. All interviews were audio taped for accurate transcription. Analysis and was done via Giorgi's method, which includes reading notes and transcripts to gain an understanding of the whole, translation to the language of science, and integration into the meaning of the study.

\section{Results}

Common themes were identified among pregnant and nonpregnant women. These themes included the following: the need for exercise, older age makes losing weight more difficult, having a routine that includes exercise/time management is important, family/social support is important, breastfeeding, role strain mom versus career, and more education on nutrition and exercise is needed from healthcare providers.

\section{Conclusion/Implications for Nursing Practice}

The themes discussed by the women who participated in this study are similar to those discussed in previous research. Though the focus of this study was women's perception of more broad factors that might be related to excessive pregnancy weight gain and postpartum weight retention, most women did not address these issues in a significant way. Even with the use of probes, women in the study still tended to go back to the individual level issues (e.g., family support, role strain, effects of older age). This may reflect the common cultural belief in the United States that emphasizes individual responsibility.

\section{The Impact of Nursing Case Management on Women With Diabetes in Pregnancy}

Corinn Taylor, MSN, RN, TriHealth, Cincinnati, $\mathrm{OH}$
Objective

T o investigate the effectiveness of nursing case management on pregnant diabetics on maternal and fetal/neonatal outcomes using the following research questions: Is there a difference in outcomes for pregnant diabetic women who receive nursing case management services versus those who do not? Is there a difference in outcomes for pregnant diabetic women who receive weekly/biweekly face-to-face visits with the 
nursing case management versus those who receive weekly/biweekly telephone nursing case management?

\section{Design}

A retrospective chart review of 93 pregnant women with preexisting diabetes and gestational diabetes mellitus (GDM) and their offspring.

\section{Setting}

A clinic setting and a private practice located within a large midwestern hospital.

\section{Patients/Participants}

Pregnant women with preexisting diabetes and GDM whose hemoglobin A1C was greater than $6.5 \%$ between January 2003 and June 2009.

\section{Methods}

Patients who received prenatal care between January 1, 2003 and June 2006 were the non-nursing case management group. Patients who received prenatal care in the private physician practice between July 2006 and June 2009 were the telephone nursing case management group. Patients who received care in the clinic between July 2006 and June 2009 were the face-to-face nursing case management group. A data collection sheet was created based upon maternal and neonatal outcomes related to diabetes in pregnancy. The independent variables were participation in either type of nursing case management. The maternaldependent variables were $\mathrm{A} 1 \mathrm{C}$, preterm labor, and preeclampsia. The neonatal/fetal variables were macrosomia, respiratory distress syndrome, Keywords and plasma glucose level at birth.

Results nursing case management diabetes pregnancy

A total of 93 pregnant women, 40 in the nonnursing case management group, 53 in the nursing case management group (31 in the face-toface nursing case management group and 22 in the telephone nursing case management group) were included in the analysis. No significant differences between groups were observed, except the $\mathrm{A} 1 \mathrm{C}$ significantly decreased $(p=.037)$ for both groups from first trimester (non-nursing case management $A 1 C=9.27[S D 2.6]$ and nursing case management $A 1 C 8.96$ [SD 1.7]) to the second trimester (non-nursing case management $\mathrm{A} 1 \mathrm{C}=$ 7.32 [SD 1.8], nursing case management $\mathrm{A} 1 \mathrm{C}=$ 7.10 [SD 1.3]), and the lower levels were sustained throughout the third trimester (non-nursing case management $A 1 C=7.16[S D$ 2.1] and nursing case management $A 1 C=6.85$ [SD 1.8]). However, the nursing case management group maintained tighter glycemic control during the second and third trimesters based upon the mean differences in $\mathrm{A} 1 \mathrm{C}$

\section{Conclusion/Implications for Nursing Practice}

Either method of nursing case management is effective in assisting pregnant diabetics improve their $\mathrm{A} 1 \mathrm{C}$, an indication of improved glycemic control, which research shows contributes to improved birth outcomes. Future research needs to be done to determine if strategies for glycemic control can be maintained long term.

\section{Uterine Rupture Among Women With Unscarred Uteri: A Case-Control Comparison}

\section{Objective}

To compare severe maternal and infant outcomes from uterine ruptures among women with unscarred uteri versus women with a prior cesarean.

Design

Matched case-control study.

Setting

Massachusetts.

Patients/Participants

Women in the Massachusetts Uterine Rupture Study with a singleton pregnancy of $\geq 20$ weeks gestation, no trauma, and labor. Cases were all women with unscarred uteri meeting criteria. Controls were randomly chosen among women with uterine rupture meeting criteria who had a prior cesarean.
Methods

All uterine ruptures in Massachusetts between 1990 and 1998 were identified using ICD-9 codes from linked hospital discharge and birth/fetal death certificate files. Uterine rupture was confirmed by medical record review. Maternal data were abstracted from maternal hospital records by trained maternity nurses. Severe maternal outcomes were defined as death, hysterectomy, FACNM, Baystate Medical transfusion, ICU admission, or assisted ventila- Center, Springfield, MA tion. Two neonatologists independently reviewed Angela Nannini, $\mathrm{PhD}, \mathrm{RN}$, infant records to identify death related to the uter- FNP, University of ine rupture and assess prognosis at discharge. Massachusetts, Lowell, Lowell, Each case was matched with two control women MA on delivery year \pm 2 years and gestational age Mary K. Barger, PhD, RN, \pm 2 weeks; $t$ tests and chi-square statistics were MPH, CNM, FACNM, used as appropriate. Conditional logistic regres- University of California, San sion generated odds ratios and 95\% confidence Francisco, San Francisco, CA intervals. 


\begin{abstract}
Keywords
uterine rupture

unscarred uterus

prior cesarean

maternal morbidity

\section{Childbearing}

Poster Presentation

Results

Of the 49 uterine ruptures in women without a prior cesarean, 36 women had unscarred uteri and met study criteria and were matched to 72 control women. Exact matches on delivery year and gestational age occurred in $85 \%$ of the controls. Case and control women did not differ on socio-demographic variables. Control women had a higher parity since they had at least one prior cesarean. Case women experienced more dilatation $\&$ curettage $(\mathrm{D} \& \mathrm{C})(p=.023)$. The two groups did not differ by hospital level, oxytocin, or epidural use.

Women with unscarred uteri were four times more likely to have severe morbidity (OR 4.19; 95\% Cl: $1.72,10.19$ ) with half requiring hysterectomy, $47 \%$ transfusion, and 33\% ICU admission.
\end{abstract}

Though both groups experienced the same percentage of individual signs of uterine rupture (pain, vaginal bleeding, nonreassuring fetal status), $58 \%$ of case women had $\geq 2$ of these signs versus $29 \%$ of controls. Of case women, $86 \%$ experienced a severe maternal morbidity. Case women's hospital stays were 2 days longer than women with a prior cesarean. Infant outcomes did not differ between groups.

\section{Conclusion/Implications for Nursing Practice}

Though uterine rupture in a woman without a prior cesarean is uncommon, when it occurs obstetric providers should be prepared for more severe maternal morbidity that may be mitigated with prompt surgical intervention and heightened hemodynamic surveillance.

\title{
Prenatal Care, Social Support, and Health-Promoting Behaviors of Immigrant Latina Women in a Disaster Recovery Environment
}

Veronica Barcelona de
Mendoza, MSN, MPH, RN,
APHN-BC, Louisiana State
University Health Sciences
Center, New Orleans, LA
Jane Savage, RN, PhD, LCCE,
Loyola University, New
Orleans, LA
Emily Harville, PhD, Tulane
University, New Orleans, LA
Gloria P. Giarratano, APRN,
CNS, PhD, Louisiana State
University Health Sciences
Center, New Orleans, LA
Keywords
Latino
disaster
pregnancy
health behaviors
social support
L

Childbearing

Poster Presentation

\begin{abstract}
Objective
The Hurricane Katrina disaster continues to mold the culture, demographics, and healthcare environment in New Orleans. Latinos have played a key role in the rebuilding of the city, arriving soon after the storm to seek employment. Immigrant Latina women face unique challenges to those who were present for the disaster in that they arrived to a devastated healthcare infrastructure and often encounter language barriers. Data are needed to describe what the long-term effects disaster recovery experiences have on maternal health and utilization of services.
\end{abstract}

\section{Design}

Cross-sectional, descriptive study.

\section{Setting}

Recruitment and data collection occurred at community prenatal care clinics, Healthy Start, and hospital prenatal classes in the Greater New Orleans area.

\section{Patients/Participants}

Prenatal women ( $n=220,24-40$ weeks gestation), currently enrolled in prenatal care and living in the Greater New Orleans area.

Methods

Measures analyzed included previous disaster exposure, perception of disaster recovery, depression (Edinburgh Depression Scale), posttraumatic stress disorder (Post-traumatic Checklist), and pregnancy-related distress (Lobel scale).
Linear regression was used to model mental health outcomes, with adjustment for race, marital status, education, employment, age, and smoking.

\section{Results}

Women interviewed were African American (70\%), White $(16 \%)$, or Hispanic (13\%). Most were unmarried, between the ages of 20 and 25 years and had an annual income of less than $\$ 15,000$. Latinas $(N=29)$ were less likely to use Healthy Start than other women $(p=.02)$ and less likely to report receiving information about birth control ( $p=.01)$, and using illegal drugs $(p=.05)$, yet there were no differences found in their reported satisfaction with prenatal care. Immigrant women who were not fluent in English were more likely to report low social support and to say they did not have someone to talk to about their problems $(p=$ .01). Latinas were less likely to smoke $(p=.02)$, less likely to exercise $(p=.01)$, and less likely to eat at least three fruits/vegetables a day.

\section{Conclusion/Implications for Nursing Practice}

Pregnant Latina women report lower utilization of prenatal services and education related to family planning. Immigrant women reported less social support and less health-promoting behaviors than other groups. Latinas are a particularly vulnerable population due to lower levels of education, potential language barriers, and less utilization of social services than other racial/ethnic groups. Health and social service provision may have to adapt to provide care to this unique population. 


\section{Effects of Full-Term Infant Massage on Mother's Emotional State}

Objective

other-infant bonding disorders affect a
mother's ability to cope with the care of her infant and can have long-term adverse consequences for the mother-infant relationship. Evidence demonstrates the benefits of infant massage with preterm and low birth weight infants to improve the mother's mental health, but benefits are unknown among mothers with full-term infants. The aim of this study is to evaluate the effects of full-term infant massage for a 4-week period on mother's emotional state.

Design

This is an intervention research.

Setting

The study was conducted in Shiga Prefecture, Japan.

Patients/Participants

Primiparous mothers at 3 months postpartum who attended an infant massage class participated in this study as intervention group $(n=20)$.

\section{Methods}

Mothers were encouraged to massage their infants for 10 to 15 minutes at least once per day for 4 weeks, starting when their infants were 3 months old. The control group was paired with the intervention group by matching the parity and postpartum period $(n=20)$, and the mother was required to hold her infant for 15 minutes instead of massage. The mother's emotional status was assessed using a Japanese version of the Profile of Mood States and Postpartum Bonding Questionnaire at the beginning and the end of the intervention. The Profile of Mood States assesses six emotional states as subscales: tension-anxiety, depression, anger-hostility, vigor, fatigue, and confusion.

\section{Results}

The mothers in the intervention group had done infant massage every day. No significant differences were observed in all subscales of Profile of Mood States and total Postpartum Bonding Questionnaire scores between the intervention and control groups at either 3 or 5 months postpartum. The score of anger-hostility at 5 months postpartum was significantly lower than that at 3 months postpartum in the intervention group but not the other subscales. A significant correlation was observed between the total Postpartum Bonding Questionnaire scores and score of tension-anxiety at 3 months postpartum $(p<.05)$, and between the total Postpartum Bonding Questionnaire scores and score of fatigue at 5 months postpartum in the intervention group $(p<.05)$, but not in the control group.

\section{Conclusion/Implications for Nursing Practice}

Through massage, the mother learns how to cope with the care of her infant and enhances her confidence in parenting abilities. This study suggests that the daily practice of full-term infant massage by the mother for 4 weeks may have beneficial effects on the maternal emotional mood.

\section{Maternal Hypothermia in Scheduled Cesarean Births and Neonatal Outcomes}

Objective o evaluate the relationship between mater-
nal hypothermia and newborn temperatures inesesarean births.

Retrospective chart review of low-risk cesareans performed over a 6-month time period using epidural anesthesia.

\section{Setting}

Urban academic medical center in the southeastern United States providing care to a diverse population of families.

\section{Patients/Participants}

Low-risk mothers at greater than 39 weeks gestation scheduled for cesareans with planned epidural anesthesia.

\section{Methods}

Retrospective chart review following Institutional Review Board approval. Statistical analysis included descriptive analysis of the sample and odds ratio.
Hiroko Watanabe, $\mathrm{PhD}$, RM, RN, Shiga University of Medical Science, Otsu, Japan

Yayoi Tanaka, RM, RN, Shiga University of Medical Science, Otsu, Japan

Shinobu Nomachi, RM, RN, MS, Shiga University of Medical Science, Otsu, Japan
Brenda Baker, MN, RNC, CNS, Virginia Commonwealth University Health System, Richmond, VA

Keywords maternal hypothermia neonatal hypothermia epidural anesthesia cesarean

\section{Childbearing}

Poster Presentation 
Keywords infant massage maternal emotional mood mother-infant bonding

\section{Childbearing \\ Poster Presentation}

Results

The sample included 143 charts. From this sample there were $46(36.5 \%)$ recorded cases of newborn hypothermia, and 27 (21\%) cases of maternal hypothermia. Using logistic regression, the odds of a newborn being hypothermic, given that the mother was hypothermic postop was 2.1 (Cl: 0.856-5.139 $p=.1055$ ). Despite being statistically nonsignificant most likely related to sample size, there is a clinical significance, as greater than one third of the sample of newborns experienced hypothermia during the transition period of birth.

Conclusion/Implications for Nursing Practice

Maternal hypothermia is clinically significant to neonatal outcomes. Nurses have the responsibility to address hypothermia in mothers undergoing cesareans through assessment and interventions pre- and postoperatively.

\section{Oxytocin Safety Measures: A Practice Team Approach Using Evidenced-Based Medicine and Electronic Documentation}

Cathy Waggoner, RNC, BSN, Freeman Health System, Joplin, MO

Keywords

Oxytocin

induction

tachysystole

\section{Childbearing}

Poster Presentation
Objective

T o standardize the use of a high alert medication, oxytocin, supported by evidence-based best practice in a community health labor and delivery unit.

Design

Tools, workflow, education, and electronic documentation were based on the 2008 National Institute for Child Health \& Human Development fetal heart rate guidelines, the Association for Women's Health, Obstetric, and Neonatal Nurses' guideline for cervical ripening and induction and augmentation of labor, and implementation of a conservative checklist-based protocol for oxytocin administration.

Patients/Participants

Perinatal Practice Committee: obstetricians, perinatologist, midwife, maternal child educator, obstetric TraceVue system administrator, nursing staff, director of maternal child, birthing center unit coordinator, risk management, and health system medical director.

\section{Methods}

The project took place at Freeman Health System Birthing Center, which provides perinatal care for 2,500+ births/year. A multidisciplinary team reviewed the literature and determined needs for evidenced-based protocols, revision of guidelines, staff education, and performance metrics. Leveraging the Philips OB TraceVue documentation system, staff could electronically document the checklists, assessments, and care provided. Data were mined this doesn't really make sense, maybe she meant "combined" into metrics reports to document progress of the program implementation. Creation of standardized oxytocin tools for nursing and medical staff included the following: Oxytocin Protocol Policy, Oxytocin Orders Revision, Induction/Augmentation of Labor Informed Consent, predelivery evaluation, PreOxytocin Checklist, In-Use Oxytocin Checklist, and Tachysystole Algorithm. Education tools were provided for patients, and all nursing and medical staff were required to complete the Education Module. To support accurate data collection, the team created electronic documentation for PreInduction Checklist, Bishop Score, and In-Use Checklist, which previously were not available to the staff.

Results

Time periods (2009-2010) were similar with approximately 400 patients with gestational age greater than 39 weeks, average hours of infusion to delivery remained at 8 hours, cesarean rate of $9 \%$ to $12 \%$, and a decrease from nine to one infant with a 5-minute Apgar score $<7$. The 2010 period had no admissions to the neonatal intensive care unit (NICU) with an estimated cost saving of $\$ 200,000$. The incidence of tachysystole dropped from $52 \%$ to $21 \%$ for all elective inductions.

Conclusion/Implications for Nursing Practice Following the guidelines and protocols Freeman Health demonstrated no significant increase in cesarean rate, a decrease in amount of oxytocin administration, and elimination of NICU admissions as a result of elective induction. Anecdotally, the team attributes the success to strong leadership, dedication, persistence, teamwork, a desire for learning, and the commitment of physician champions. The Freeman Health project demonstrates that using teamwork, evidence-based protocols, and electronic documentation can lead to safer patient care. 


\section{Description of Mother Comorbidities and Infant Outcomes by Entry into Prenatal Care}

\begin{abstract}
Objective
$D$ renatal care has shown to improve infant outcomes. The current standard is entry into prenatal care $<14$ weeks after conception. The purpose was to evaluate comorbid conditions present in mothers with no, standard, and late entry into prenatal care and differences existing among infants based on mother's prenatal care status.

Design

Prospective comparative descriptive design with consecutive sampling was used to describe differences in maternal comorbid health conditions and infant outcomes in mothers with no prenatal care, early prenatal care ( $\leq 14$ weeks), or late prenatal care (>14 weeks). The study was approved by the Institutional Review Board.
\end{abstract}

\section{Setting}

Midwest nonteaching community medical center.

Patients/Participants

Medical records of 655 mothers and 703 delivered infants.

\section{Methods}

We evaluated mothers and births $\geq 20$ weeks gestation or $<20$ weeks gestation with signs of life at birth regardless of infant outcomes within a 6month study period. Data were collected as part of normal documentation and retrieved postdelivery. Descriptive statistics and analysis of frequency data were performed using Statistical Package for the Social Sciences.
Results

Of the participants, 522 mothers received early care, 136 mothers received late care, and 7 mothers received no care. The sample included 571 White, 56 African American, 15 Hispanic, and 23 other ethnicity mothers, comparing favorably to the regional population estimates. Mothers with early care were significantly older than mothers with late care. The mean gravida/para of mothers with late care was significantly higher than with early care. Mothers with late or no care reported significantly greater use of state funded Medicaid than with early care. Mothers with no care had a significantly higher body mass index as compared with early and late care. Mothers with early care had significantly less report of drug abuse than with late care. Mothers receiving late care had more infants who were admitted to the neonatal intensive care unit compared with early care. A significantly higher percentage of mothers with no care delivered by vaginal birth after cesarean compared with early and late care. Mothers with no care compared to early and late care were found to have a significantly higher incidence of gestational diabetes. Oligohydramnios occurred more often in those with late care, and no statistically significant differences were found for the infant outcome variables among groups.

Conclusion/Implications for Nursing Practice

Prenatal care has shown to be important in infant outcomes; however, no statistically significant differences in infant outcomes were identified in this study.

\section{Noncoached Pushing in the Second Stage of Labor}

Objective

T he literature indicates that noncoached pushing during the second stage of labor may be beneficial to the mother and fetus. Yet most of the research is conducted on women with no epidural. The most common practice at St. Luke's Hospital, as well as elsewhere, is coached pushing, where the patient is told to push three times during each contraction, for 10 seconds each. We aimed to understand factors that influence the use of noncoached pushing, which encourages the woman to listen to her body and push the way she wants and to determine if noncoached pushing for patients with an epidural is feasible. We further examined the relationship between noncoached pushing and several outcome variables.
Design

Angela Balogach, RN, BSN, St. Quasi-experimental research study using a con- Luke's Hospital, Bethlehem, venience sample of laboring women.

Patients/Participants

Laboring women with singleton pregnancies who reached the pushing stage of labor.

\section{Methods}

Participants were classified as either "coached"
PA

Cassandra R. Althauser, RN, BSN, MPH, St. Luke's Hospital, Bethlehem, PA

Melanie Martin, RN, BSN, St. Luke's Hospital, Allentown, PA

Susan Walp, RN, St. Luke's

Hospital, Bethlehem, PA

Hospita or "noncoached." The influence of parity, use of oxytocin, epidural, time spent laboring down, urge to push, and time spent pushing were initially examined via chi-square analysis, and multivariate direct logistic regression was conducted to determine relationships with episiotomies, operative
Michele A. Donovan, RN, BSN, St Mary's Medical Center, Evansville, IN 
Keywords

noncoached pushing second stage of labor epidural

perineal laceration

\section{Childbearing}

Poster Presentation deliveries, perineal lacerations, and Apgar scores. Additionally, nurses were interviewed about why they used noncoached or coached pushing. Education was provided for nurses and doctors at seminars regarding the research behind the use of noncoached pushing, and its use was encouraged.

\section{Results}

The use of noncoached pushing was related to multiparity, labor progression without the use of oxytocin, labor without an epidural, an urge to push at $10 \mathrm{~cm}$, and a shorter time spent pushing $(p<.05)$. In bivariate relationships, noncoached pushing is related to a decrease in episiotomies, operative deliveries, and perineal lacerations $(p<.05)$. Multivariate logistic regression revealed a trend in the data suggesting that noncoached pushing is related to fewer third- and fourth-degree lacerations, even when controlling for other factors $(p<.1)$. In women with epidurals, multivariate logistic analysis revealed a significant relationship between coaching and operative deliveries $(p<.05)$. A common theme in the interviews with nurses was that women who were not pushing effectively were coached during their pushing.

Conclusion/Implications for Nursing Practice

Noncoached pushing during labor may have maternal benefits. Though women without an epidural were more likely to receive noncoached pushing, more than half of women with an epidural still had noncoached pushing. This indicates that having an epidural is not prohibitive of noncoached pushing.

\section{The Relationship Among Maternal-Infant Bonding, Spirituality, and Maternal Perception of the Childbirth Experience}

Linda K. Bennington, BS, MS, Objective

BSN, MSN, PhD, Old

Dominion University, Norfolk, VA

Keywords

maternal-infant bonding perceived birth experience patient advocacy spirituality

\section{Childbearing}

Poster Presentation
The beginning of life is an intense experience for mother and baby and sets the foundation for future interactions. Researchers have theorized that maternal-infant bonding begins prenatally and continues on through the postnatal period. Researchers examined that process to determine if prenatal bonding was related to postnatal bonding and found only a modest correlation. The objective of this study was threefold: (a) Examine the relationship between a woman's perceived birth experience and maternal-infant bonding; (b)

Examine the relationship between spirituality and maternal-infant bonding; (c) Examine the relationship between perceived birth experience, spirituality, and maternal-infant bonding.

\section{Design}

This was a mixed design using quantitative and qualitative methods.

\section{Setting}

Women were recruited for an Internet survey through various childbirth websites, nurses' associations, and perinatal listserv communications.

Patients/Participants

A total of 402 women responded to the online survey.

\section{Methods}

Sixty-seven items in three instruments: Perception of Birth Scale; Spirituality; and Maternal Attachment Inventory were to be completed and comments could be left as desired. Approximately 300 participants finished the survey completely and these responses were used in the analyses.

\section{Results}

More than 190 participants provided extensive comments regarding their experiences. Predictive Analytical Software (PASW 18) was used to analyze data and correlations were run on the measurements of the three instruments as well as a regression analysis. The perceived birth experience had the strongest correlation to maternalinfant bonding and was found to have a stronger influence on bonding as well.

\section{Conclusion/Implications for Nursing Practice}

Since the perceived birth experience was found to have the strongest correlation to bonding, we need to examine the myriad of interventions that are currently being used. Implications for nursing practice include being more of an advocate for the laboring woman; facilitating bonding immediately after birth; and discussing need for interventions honestly and openly with the laboring woman and her partner. 


\section{Effectiveness of Intradermal Sterile Water Injections in Control of Lower Back Pain in Latent and Active Labor}

\author{
Objective \\ To \\ o determine the effectiveness of intradermal \\ sterile water injections for lower back pain in \\ latent and active stage of labor.
}

\section{Design}

Randomized controlled trial approved by the Institutional Review Board.

\section{Setting}

Labor and Delivery unit with 10 beds in a rural Idaho hospital.

\section{Patients/Participants}

Adult laboring women at or greater than 37 weeks gestation with a low-risk pregnancy admitted to the labor and delivery unit at $3 \mathrm{~cm}$ dilation or less.

\section{Methods}

Participants were assigned to one of two intervention groups: Group A (one set of sterile water injections in latent labor) or Group B (one set of sterile water injections in latent labor and one set of sterile water injections in active labor). The pain relief is evaluated using the Visual Analog Scale before the intervention then at set intervals after Jessica Hockenberry, RN, St. the intervention. Luke's Magic Valley Regional Medical Center, Twin Falls, ID

\section{Results} do date there are 12 patients enrolled in the study. Luke's Magic Valley Regional Of the 12, six complained of back pain and re- Medical Center, Twin Falls, ID ceived the injections (Group A: $n=3$ and Group B: $n=3$ ). All six patients received the first set of injections at $3 \mathrm{~cm}$ dilation or less. None of the patients received the second set of injections at $4 \mathrm{~cm}$ or greater because of delivery or receiving an epidural. Thus, there have been no injections administered at $4 \mathrm{~cm}$ or greater to evaluate the intervention in active stage labor. Though the preliminary data suggest that the intervention is effective in latent stage labor ( $3 \mathrm{~cm}$ or less), the research team continues to collect data to complete the study objective.

\section{Conclusion/Implications for Nursing Practice}

The outcome of this study will help determine if this is an effective means of lower back pain relief that can be administered during latent and active phase labor. The results will help clinicians with the challenge of relieving the back pain a laboring woman may experience.

\section{The Contribution of Violence to Pregnancy-Related Deaths in Virginia}

\section{Objective \\ To review all deaths of women occurring dur- to determine the prevalence of the association be- tween pregnancy with violent deaths.}

\section{Design}

Virginia's Maternal Mortality Review Team is a multidisciplinary team that reviews all deaths of Virginia residents who were pregnant when they died or who died within 1 year of pregnancy regardless of the outcome of the pregnancy or the cause and manner of death, termed "pregnancy-associated death." The Maternal Mortality Review Team determines if each death was pregnancy-related and whether systems changes could be instituted to avert similar deaths. A pregnancy-related death is a death resulting from complications of the pregnancy itself; the chain of events initiated by the pregnancy that led to death; or aggravation of an unrelated condition by the pregnancy that subsequently causes death. The team determines if the death was pregnancy-related based on the cir- Victoria M. Kavanaugh, PhD, cumstances surrounding each death. Homicides Virginia Department of Health, and other violent deaths are characterized as Richmond, VA pregnancy-related using the criteria that the chain Faith M. Miller, MSN, RN-BC of events initiated by the pregnancy led to death, OB-BC, WHNP-BC, Bon for example, through review of case facts that re- Secours St. Mary's Hospital, vealed the perpetrator of homicide was spurred to Richmond, VA commit the homicide because of the pregnancy Keywords or death investigation of a suicide revealed that pregnancy-related death the impetus was the pregnancy through notes referencing the pregnancy or through interviews with persons close to the victim. This study reports on cases that were determined to be directly related to the pregnancy and describes the causes and manners of death.

Setting

Retrospective record review.

Patients/Participants

All women who died while pregnant or within year of pregnancy. 
Methods

Multiple case studies of the population of women who died within 1 year of pregnancy in a single state over a 5-year period.

Results

Of the 210 pregnancy-associated deaths occurring from 1999 to 2003, 86 cases (40.9\%) were determined to be pregnancy-related. Leading causes and/or manner of pregnancy-related death were cardiovascular disorders (15.1\%, $n=13)$, pulmonary embolism $(12.8 \%, n=11)$, cardiomyopathy $(12.8 \%, n=11)$, hemorrhage $(9.3 \%, n=$
8), homicide $(8.1 \%, n=7)$, and suicide $(7.0 \%$, $n=6)$. More than one in five $(22.1 \%)$ pregnancyrelated deaths resulted from violence. All but one violent death was considered to be preventable through changes in policy or practice.

Conclusion/Implications for Nursing Practice Pregnancy may serve as a catalyst for violent death through several mechanisms. Reviewing deaths occurring during or near pregnancy reveals the magnitude of the problem and provides an opportunity to develop prevention and intervention strategies to reduce these deaths.

\section{Perinatal Mortality After Hospital Admission Among Planned, Out-of-Hospital Births, 2004-2008}

Janice E. Snyder, RNC, OHSU, Objective

Portland, OR

Teresa T. Goodell, PhD., RN, CNS, CCRN, APRN, BC OHSU, Portland, OR

Lani Doser, RN, BSN, MN OHSU, Portland, OR

Keywords

planned home birth hospital transfer out-of-hospital birth

\section{Childbearing Poster Presentation}

T

\section{Design}

\section{Setting} describe the incidence of perinatal death (defined as intrauterine fetal death at $\geq 28$ weeks gestation or neonatal death at $\leq 28$ days of age) in a population of hospital admissions from planned out-of-hospital births.

Retrospective review of admissions to labor and delivery and neonatal intensive care unit in one urban hospital from January 1, 2004, to December 31, 122008.

Academic tertiary medical center.

\section{Patients/Participants}

Chart review of patients meeting study criteria.

\section{Methods}

Antepartum and intrapartum hospitalizations from planned out-of-hospital births were included if they resulted in birth at study hospital. Maternal postpartum and/or neonatal hospital admissions were included if occurring $\leq 24$ hours after planned out-of-hospital delivery. Eligible cases were reviewed and data entered into electronic database.

\section{Results}

The total number of transfers represented 223 pregnancies, including six with twin gestation for a total $N=229$ births. Transfer occurred at a variety of time points: antepartum, $n=31$; intrapartum, $n=147$; postdelivery maternal and neonate, $n=$ 7; postpartum maternal only, $n=12$; neonate only, $n=26$. Live birth status was not available for six neonates in cases of maternal only or neonatal only transport. In 223 births with mortality data, eight deaths were characterized as follows: intrauterine fetal demise before 37 weeks gestation, $n=3$; intrauterine fetal demise on or after 37 weeks gestation, $n=2$; neonatal death within 7 days after birth, born at term, $n=2$; neonatal death at age 8 to 28 days, born at term, $n=1$.

The combined fetal and neonatal mortality rate was $8 / 223(3.59 \%)$, yet comparison with available vital statistics requires a denominator that can account for total regional planned out-of-hospital births as well as area hospital transfers. One of the eight deaths was due to lethal congenital anomalies. Among the remaining seven, at least one of the following risk factors was present: preeclampsia or gestational hypertension, postdates gestation, or planned out-of-hospital vaginal breech delivery.

Conclusion/Implications for Nursing Practice It is difficult to assess the safety of planned home birth in most of the United States because planned out-of-hospital births with hospital transfers are not identifiable by vital records. In Oregon, the number of out-of-hospital births increased from $2.2 \%$ of total births $(1,003 / 46,453)$ in 2004 to $2.9 \%$ $(1,431 / 49,492)$ in 2008 . In the county where the study hospital is located, there were 11,027 total births, which includes 365 planned out-of-hospital births and 10 unplanned out-of-hospital births in 2008 (3.3\% planned out-of-hospital births). These data underscore the imperative for comprehensive and prospective information on this population. 


\section{Assessment of an Alternative Stabilization Device for Electronic Fetal Monitoring Utilizing External Ultrasound and Tocodynamometer in Labor and Delivery Patients}

Objectivas

o assess whether an alternative stabilization

device for external electronic fetal monitoring such as the Pumpkin Patch can provide safety, comfort, and tracing data for interpretation that favorably compare to the circumferential belt system currently in use during labor and delivery.

\section{Design}

A prospective, randomized trial comparing the Pumpkin Patch to the traditional circumferential belt.

\section{Setting}

Labor and delivery unit in an academic medical center.

\section{Patients/Participants}

A total of 30 pregnant women admitted to labor and delivery who satisfy all of the following eligibility requirements will be asked to participate: 18 years or older; at term (37-42 weeks gestation); singleton pregnancy; and no known allergy to adhesives. Aside from those not meeting the above requirements, any woman meeting one or more of the following criteria will be excluded: highrisk/critically ill pregnancy; patient declines consent; body mass index greater than 30; elective repeat cesarean.

\section{Methods}

A Nurse Assessment Survey and a Patient Satisfaction Survey will be utilized. Analysis will include comparing the average number of required nurse monitor adjustments per hour and the nurse expe- rience using the Nurse Assessment Survey; $t$ test Frann Teplick, MSN, RN-BC, will be utilized. A Patient Satisfaction Survey will CNS, University of California be utilized to assess the patient experience.

San Diego Medical Center, San Diego, CA

Results

Expected results include the following:

- Adjusting the Pumpkin Patch device will be at least equivalent to the circumferential belt; it is hoped that data will show less adjustments are needed with the Pumpkin Patch device. Therefore, lower adjustment frequency corresponds with higher device efficacy.

- The Nurse Assessment Survey Likert score medians will be higher for the Pumpkin Patch group.

- The Patient Satisfaction Surveys will show higher scores for the Pumpkin Patch device.

Conclusion/Implications for Nursing Practice

This research will allow us to identify another option for maintaining external electronic fetal monitoring devices for women entering labor and delivery. The proposed benefits of this device for the patient include improved stabilization of external ultrasound and tocodynamometer monitors and increased comfort during labor. For the healthcare staff, the device may decrease the time spent adjusting the EFM apparatus compared to the current standard and also improve the accuracy of fetal heart rate tracing interpretation. This may be especially beneficial during procedures performed while the patient is wearing the ultrasound and tocodynamometer monitors (e.g., epidural administration, restroom use, etc.). 


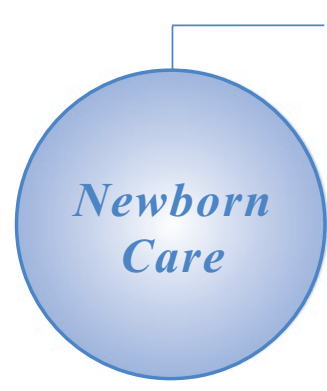

Rita H. Pickler, PhD, RN, PNP-BC, FAAN, Cincinnati Children's Hospital Medical Center, Cincinnati, OH

Heather Tubbs-Cooley, PhD, $\mathrm{RN}$, Cincinnati Children's Hospital Medical Center, Cincinnati, $\mathrm{OH}$

Sharon Cone, MS, RN, NNP, Virginia Commonwealth University, Richmond, VA

Jacqueline McGrath, PhD, RN, FNAP, FAAN, Virginia Commonwealth University, School of Nursing, Richmond, VA

Paul Wetzel, PhD, Virginia Commonwealth University, Richmond, VA

Al Best, PhD, Virginia Commonwealth University, Richmond, VA

Marty Lewis, BS, RN, Virginia Commonwealth University, Richmond, VA

Barbara Reyna, PhD, RN, NNP-BC, VCU Health System, Children's Hospital of Richmond, Richmond, VA

Keywords

NICU design preterm infant feeding caregiving environment

\section{Effects of Neonatal Intensive Care Unit Environmental Characteristics on Preterm Infant Oral Feeding}

Objective

$\mathrm{M}$ any neonatal intensive care units have been redesigned from multipatient wards to single family room design with the expectation of improved patient outcomes. In this study, we examined the effect of neonatal intensive care unit (NICU) environmental characteristics (light, sound, activity) associated with wards and single family rooms on preterm infant oral feeding outcomes.

\section{Design}

This descriptive analysis was part of a larger randomized controlled trial of preterm infant feeding. The study had Institutional Review Board approval.

\section{Setting}

Data were collected in a Level III NICU that moved from a single 32-bed ward to a 30-single family room design midway through the study.

\section{Patients/Participants}

The observations involved 87 preterm infants (43 male; mean birth weight $1.4 \mathrm{~kg} ; 70 \%$ Black, nonHispanic).

\section{Methods}

Light, sound, and activity were recorded at every feeding for 14 days for 87 preterm infants resulting in 10,913 observations $(5,111$ ward, 5,802 single family room). A rating scale (1-5; $1=$ lowest level) was used to record light, sound, and activity. Oral feeding outcome was the proportion of prescribed breast milk or formula consumed by nipple at each scheduled feeding. Data were analyzed with descriptive statistics and chi-square analysis.

\section{Results}

Light, sound, and activity were rated significantly lower in the single family rooms versus the ward ( $p$ $<.0001$ for all measures). Feeding times of 9:00 a.m., 12 noon, 3:00 p.m. were associated with the highest levels of light, sound, and activity in both unit designs, with the ward design significantly higher $(p<.001)$. Infant oral feeding was significantly affected by infant postconceptual age, morbidity, light, and time of feeding.

\section{Conclusion/Implications for Nursing Practice}

Preterm infant feeding is affected by many things, including characteristics of the infant that may not be amenable to intervention. However, oral feeding is also influenced by environmental characteristics that can be changed. We found that infant oral feeding was significantly improved by lower light levels. Though it is acknowledged that unit design may affect caregivers' ability to alter light levels, a variety of strategies do exist, including feeding infants in specially designated feeding rooms, retrofitting units with bedside task lighting, and using overhead shields to reduce light exposure during feeding. Time of feeding also affected outcomes, and the busiest daytime hours had a significantly negative impact on oral consumption. Some unit practices may contribute to this environmental characteristic, including bedside rounds, clustering of care, and multiple assessments conducted within a short time interval. These activities need further study to determine their impact on important health outcomes.

\section{Does Skin-to-Skin Contact at Birth Really Make a Difference in Exclusive Breastfeeding Rates at Discharge?}

Pamela S. Mellin, RN, MSN, APNC, Morristown Medical Center - Atlantic Health, Bernardsville, NJ

\author{
Objective \\ The benefits of breastfeeding are well known. \\ The Surgeon General's Call to Action to Sup- \\ port Breastfeeding highlighted the impact of ma- \\ ternity care practices on the establishment of ex-
}

clusive breastfeeding. The Centers for Disease Control identified placing the newborn skin-to-skin with the mother after birth as a breastfeeding supportive practice. Our healthcare system reviewed the maternity care practices at our hospitals and 
decided to implement skin-to-skin contact at birth. Would skin-to-skin contact at birth really make a difference in the exclusive breastfeeding rates at discharge?

\section{Design}

An education program on the importance of skinto-skin contact after birth was given to the nursing staff. Education included definitions and benefits of skin-to-skin contact and a description of its role in successful breastfeeding. The nursing staff was instructed to offer skin-to-skin at every vaginal delivery.

\section{Setting}

A sample of convenience of women giving birth at Atlantic Health hospitals in northwestern New Jersey. Morristown Medical Center is a regional perinatal center with approximately 4,000 births annually. Overlook Medical Center is a perinatal intensive hospital with approximately 2,400 births annually.

\section{Patients/Participants}

Breastfeeding women who gave birth vaginally to a term singleton infant. We excluded cesarean and preterm birth, multiple gestations, and teen mothers. A sample size of 148 was required at each hospital for the skin-to-skin group and the control group for a 95\% confidence interval.
Methods

Retrospective closed chart review comparing the exclusive breastfeeding rates at discharge of women who did not have skin-to-skin contact prior to implementation of skin-to-skin and women who did have skin-to-skin contact with their infants. Exclusive breastfeeding was defined as no other liquid or solid fed to the infant except for medication.

Results

Data were compared using paired $t$ tests. A $p$ value of less than .05 was considered significant. At Morristown Medical Center the exclusive breastfeeding rate prior to the implementation of skin-to-skin was 54\%. The exclusive breastfeeding rate for women with skin-to-skin at birth was $74 \%$. This resulted in a $p$-value of .0003 , which was statistically significant. At Overlook Medical Center the exclusive breastfeeding rate prior to the implementation of skin-to-skin was $51 \%$. The exclusive breastfeeding rate for women with skinto-skin contact at birth was $63 \%$. The resultant $p$ value was .0196, which was also statistically significant.

\section{Conclusion/Implications for Nursing Practice}

Skin-to-skin contact at birth increased the exclusive breastfeeding rates at discharge for these participants. There may be increased nurse and patient satisfaction. Further research is needed.
Donna T. Poplawski, RNC

MSN, NP, Atlantic Health Morristown Medical Center, Morristown, NJ

Nancy DeFreest, RN, MA, Overlook Medical Center, Summit, NJ

Kathy Massler, RN, Overlook Medical Center, Summit, NJ

Amy Gole, RNC, EdM, IBCLC, Morristown Medical Center - Atlantic Health, Morristown, NJ

Keywords breastfeeding

skin-to-skin

exclusive breastfeeding

\section{Newborn Care}

Paper Presentation 
through weekly phone calls for 3 months, then monthly until the mother weaned or reached 6 months.

Results

At the conclusion of the program $100 \%$ of the participants met their goals, $73 \%$ were still breastfeeding at 6 months as compared to $38 \%$ in the base-line group.
Conclusion/Implications for Nursing Practice

The provision of structured telephone support decreased the risk of early weaning and empowered participants to overcome challenges and meet their stated goals. Nurses have an ethical responsibility to advocate for breastfeeding support programs that extend beyond the immediate postpartum period. Support should include provisions that enhance and sustain the woman's breastfeeding efforts. Additional research and evidence-based projects are needed to actualize the Healthy People 2012 breastfeeding objectives.

\section{Premature Infant Transition to Effective Breastfeeding: A Comparison of Four Supplemental Feeding Methods}

Susan J. Garpiel, RN-C, MN, IBCLC, Covenant Healthcare System, Saginaw, MI

\begin{tabular}{l} 
Keywords \\
breastfeeding \\
premature infant \\
supplementation \\
nasogastric feeding \\
\hline
\end{tabular}

Newborn Care Paper Presentation
Objective

T his prospective study is the first to compare four supplemental feeding methods to provide evidence regarding the optimal method to facilitate breastfeeding preterm infants.

Design

Randomized controlled trial.

\section{Setting}

Community hospital with a Level III neonatal intensive care unit (NICU) in the midwest United States.

\section{Patients/Participants}

One hundred thirty-two premature infants, ranging from 26 to 36 weeks gestation at birth were randomized into one of the four supplemental feeding method groups.

\section{Methods}

The methods investigated were nasogastric tube with pacifier, bottle with preterm nipple, cup feeding with a 30-ml medicine cup, and the Haberman infant feeder (Medela). The primary hypotheses were that there would be no significant differences in breastfeeding ability at discharge and infant tolerance to the supplementary method. Data collected on other outcome measures included breastfeeding rate at discharge, 2 and 4 weeks post-hospitalization, average daily weight gain, hospital length of stay, frequency of skin-toskin care and breastfeeding sessions, and maternal satisfaction with feeding method.

Results

The null hypothesis was rejected for both primary outcome measures. Results showed that prema- ture infants in the nasogastric tube with pacifier method had significantly better breastfeeding ability at discharge $(p=.04)$. Infants in that feeding group breastfed significantly more frequently than infants in the bottle group $(p=.01)$. Infants in that feeding group breastfed significantly more frequently than infants in the bottle group $(p=.01)$. Infants in the bottle-supplemented group demonstrated a reduction in breast milk intake per pre/postbreastfeeding test weights after the supplemental bottle was introduced. Consequently, the mothers reduced their breastfeeding frequency believing that it would expedite their infants' hospital discharge. There were no significant differences in hospital length of stay among the four methods. Furthermore, infants tolerated the nasogastric with pacifier feeding method better than the bottle group. Bottle-supplemented infants had almost four times the number of apnea, bradycardia, and oxygen desaturation events during the feeding. The variables of frequency of skin-toskin and breastfeeding sessions were positively correlated with breastfeeding ability at discharge. Higher levels of breastfeeding ability at discharge predicted continued breastfeeding at 4 weeks post-discharge.

Conclusion/Implications for Nursing Practice

The results of this study showed that supplementing the premature infant by nasogastric tube with a pacifier (when the breastfeeding mother is unable to be at the bedside for feeding) significantly supports breastfeeding ability by hospital discharge and continued breastfeeding to 4 weeks postdischarge. 


\section{Maternal Responsiveness: Early Observation of Mothers Who Bottle-Feed Is Needed in Reducing the Risk of Childhood Obesity}

\begin{abstract}
Objective
aternal responsiveness to infant communication or cues to stop or start feeding begins as early as birth. Despite continued efforts to increase the number of mothers who breastfeed their infants to age 6 months, a majority bottle-feed. Because formula-fed infants tend to gain more weight than breastfed infants, mothers who bottle-feed need to be encouraged to allow infants to self-regulate. An infant self-regulates caloric intake by giving hunger and satiety cues. The objective of this study was to measure the association between maternal responses to infant feeding cues and feeding method in 129 lowincome, mother-infant dyads in two Midwestern States.
\end{abstract}

\section{Design}

Secondary analysis of baseline data from Healthy Babies through Infant Centered Feeding, an ongoing longitudinal study, was used for this study.

\section{Setting}

Mother-infant feeding interactions were videotaped in the mother's home by trained data collectors.

\section{Patients/Participants}

Maternal participants were $\geq 18$ years, eligible for Special Supplemental Nutrition Program for Women, Infants, and Children with no known chronic illness. Infants were $<4$ months of age, had no known eating problems, and birth weight $>2,500 \mathrm{~g}$.

\section{Methods}

The maternal-infant feeding interaction was scored by two certified research assistants us- ing the Parent-Child Interaction Tool for Feed- Lisa Singleterry, MSN, BSN, ing (NCAST-F/PCI-F) and validated by consensus. RN, Michigan State University, Maternal responses were measured with the 16- Hastings, MI point caregiver contingency subscale (NCAFSCC scale) of the NCAST-F/PCI-F tool.

Results

Statistics were computed using Statistical Package for the Social Sciences 17. Mothers' ages ranged from 18 to 42 years, with $56 \%$ selfidentified as African American, 42\% White, and $30 \%$ Hispanic. Infants ranged in age from 1 to 21 weeks; $51 \%$ were male; $73 \%(n=94)$ of infants were observed bottle feeding. The mean NCAFSCC score was 8.48 (16 possible). One-way analysis of variance showed that mean scores for bottle feeding (7.9) and breastfeeding (10) were significantly different $(F=18.26, p=.000)$. Mothers who bottle-fed had lower scores on the NCAFS$\mathrm{CC}$ than breastfeeding mothers.

Conclusion/Implications for Nursing Practice In this sample the mean NCAFS-CC score was below the expected norm of 12. Nurses play a pivotal role in identification, development, and delivery of interventions to foster maternal responsiveness. Maternal responses to infant hunger and satiety cues are foundational in promoting healthy eating habits and reducing childhood obesity. Observation of maternal responsiveness and help with cue recognition in bottle feeding mothers needs to begin at birth. Further research is needed in early infancy to help bottle feeding mothers develop responsive behavior to their infants' hunger and satiety cues.

\section{Perception of Insufficient Milk in a WIC Sample}

\section{Objective}

O ur long-term goal in the United States is to improve breastfeeding exclusivity and duration rates for low-income women receiving services from the Special Supplemental Nutrition Program for Women, Infants, and Children (WIC). Women who receive WIC services breastfeed their infants for significantly shorter durations, and improving their breastfeeding duration is a national priority. Many women cite the perception of in- sufficient milk as the reason for early cessation. Sandi Tenfelde, PhD, RN, However, the association between perception of WHNP-BC, Loyola University insufficient milk and socioeconomic and demo- Chicago, Neihoff School of graphic variables for low-income women has not Nursing, Maywood, IL been addressed in the literature. The purpose of this descriptive-correlational study was to examine maternal and infant factors related to the reason for discontinuing breastfeeding for WIC participants. 
Keywords

breastfeeding

perception of insufficient milk WIC

Newborn Care
Paper Presentation

\section{Patients/Participants}

Two hundred thirty-nine WIC participants who initiated breastfeeding.

Methods

Selected components of the Interaction Model of Client Health Behavior guided the inclusion of potential predictors related to the reason for discontinuing breastfeeding. The authors used descriptive statistics and logistic regression to analyze how maternal and infant characteristics (demographic, social, health-related, environmental, motivational, cognitive, and affective) influenced the reason for breastfeeding cessation.

\section{Results}

The duration of breastfeeding was low, with an estimated $31 \%$ continuing to breastfeed at 6 months and $6 \%$ continuing to breastfeed at 12 months. Of the 239 women in this sample who provided a reason for discontinuing breastfeeding, 43\% ( $n$ $=111$ ) reported perception of insufficient milk as the reason. The majority of women who reported perception of insufficient milk did so within the first 3 months of breastfeeding (55\%). Women who reported perception of insufficient milk were more likely to be of Hispanic descent. Additional analysis is on-going.

Conclusion/Implications for Nursing Practice

Though WIC women initiate breastfeeding, the national recommendation for breastfeeding duration is not being met in this population. Additionally, many women stop breastfeeding early due to perception of insufficient milk. Findings from this study will be used to develop and test interventions to reduce the incidence of perception of insufficient milk and increase breastfeeding duration in this vulnerable population.

\section{Neonatal Head Cooling: Implications for Community Hospital Nurses}

Jennifer Ann Novack, MSN, RN, CNS, APN, Bayhealth Medical Center, Dover, DE

Melody J. Wireman, MSN, RNC, CNS, APN, Bayhealth Medical Center, Dover, DE

Robin Lynn Underwood, MSN, RNC, CNS, APN, Bayhealth Medical Center, Dover, DE

Keywords

hypoxic ischemic

encephalopathy

head cooling

neonatal outcomes

\section{Newborn Care Poster Presentation}

Objective

etermine contributing factors to one organization's high rate of neonates requiring head cooling treatment for hypoxic ischemic encephalopathy (HIE). Design and implement strategies to reduce rate by $50 \%$.

Background/Significance of Problem

Though Bayhealth Medical Center delivered 18\% of all live births in 2009 and 2010, 50\% of all neonates requiring head cooling treatment for hypoxic ischemic encephalopathy were from this organization.

\section{Design}

Retrospective chart review and qualitative interviews with labor and delivery nurses assigned to women whose neonates required head cooling for hypoxic ischemic encephalopathy.

\section{Patients/Participants}

All (100\%) neonates from May 2009 when head cooling at level III referral center began through January 2011. Inclusion criteria: neonate referred to level III neonatal intensive care unit for head cooling. Exclusion criteria: none

Methods

An audit tool with 250 different data elements and a formal interview tool were developed. Clinical nurse specialists audited all charts that met inclusion criteria. Charts that met specific red flag indicators were audited by independent physician consultants.

\section{Implementation Strategies}

Targeted hands-on nursing education for all labor and delivery nurses that included fetal strip interpretation, intrauterine resuscitation, oxytocin protocols, difficult conversation communication techniques, use of consistent National Institute for Child Health \& Human Development language when interpreting fetal strips, prompt recognition of neonates that may meet head cooling, and policy review. Additional electronic "nursing perinatal bundle" education included managing shoulder dystocia, operative vaginal delivery, advanced fetal assessment and monitoring, and Situation, Background, Assessment, Recommendation and Readback techniques.

\section{Results}

During the past 8 months there have been zero neonates that have required head cooling treatment for hypoxic ischemic encephalopathy.

\section{Conclusion/Implications for Nursing Practice}

Nursing ownership and nurse-driven interventions dramatically improve patient outcomes. 
Utilizing evidence-based practices and a systematic performance improvement process can facilitate achieving quality patient outcomes. Nurses at community hospitals have tremendous impact on neonates that qualify for head cooling due to hypoxic ischemic encephalopathy. Future plans include developing a multidisciplinary perinatal safety team at the organization that includes medical staff, nursing staff, and ancillary departments to improve maternal and neonatal outcomes.

\section{Breastfeeding Frequency During the First 24 Hours of Life for the Normal Newborn}

\section{Objective}

rofessional organizations recommend breastfeeding at least eight times in 24 hours. However, it is unclear whether this recommendation applies to the first 24 hours after birth. Nevertheless, institutional policy follows the recommendation of feeding every 3 to 4 hours. For a variety of physiologically adaptive behaviors, breastfed infants feed less frequently during the first 24 hours of life than the formula-fed infants. There is a concern over what is the normal number of feeding episodes during the first 24 hours after birth. This study was conducted to determine breastfeeding frequency and infant elimination patterns during the first 24 hours of extrauterine life in an environment with minimal medical intervention.

\section{Design}

Retrospective, descriptive study design.

\section{Setting}

A freestanding, nonprofit birth center in Northeastern Ohio.

\section{Patients/Participants}

Healthy mothers who were 18 years and older with uncomplicated vaginal birth, clear amniotic fluid, and chose to breastfeed in 2009 and 2010. Infant inclusion criteria included singleton, full-term, 5minute Apgar score $>7$, no apparent facial deformity/malformation, and no identified medical problems.
Methods

Institutional Review Board approval and letter of cooperation from the birth center were obtained. A research data collection tool was developed and used for the study.

\section{Results}

A total of 110 randomly selected charts were reviewed. On average, mothers were 28.9 years old, had 4.9 pregnancies, and had given birth 3.3 times prior to the current birth; their infants weighed 3,556 $\mathrm{g}$ at birth. On average, first breastfeeding occurred 1 hour postdelivery, and 8.2 breastfeeding sessions (SD: 1.4, range: $5-12$ ) took place within the first 24 hours. More than $50 \%$ of the infants did not receive any supplement (e.g., formula, glucose water). Within the first 24 hours, the infants voided 2.6 times (SD: 1.6, range: $0-8$ ) and had 3.5 bowel movements (SD: 1.6, range: 1-8).

\section{Conclusion/Implications for Nursing Practice}

Infants born to mothers in an environment with minimal medical intervention (e.g., no epidural anesthesia, no prolonged intravenous fluids, and no induction) breastfed on average eight times and eliminated three times in the first 24 hours after birth. Before definitive conclusion can be drawn about what would be considered the normal behaviors of breastfed infants in the first 24 hours of life, prospective studies are needed to examine the number and quality of breastfeeding sessions in other minimal medical intervention facilities.
Marie A. Cobb, MSN,

RNC-OB, IBCLC-RLC, The

University of Akron, College of

Nursing, Akron, $\mathrm{OH}$

Sheau-Huey Chiu, PhD, RN, The University of Akron,

College of Nursing, Akron, $\mathrm{OH}$

\section{"Mom, I'm Yellow": An Evidence-Based Initiative to Reduce Risks of Newborn Hyperbilirubinemia and Hospital Readmissions}

Objective

A 3-year review of newborn readmissions identified hyperbilirubinemia as the predominant diagnosis. Our objective was to improve care/processes to reduce newborn risks and prevent readmissions. An interdisciplinary Marianne Allen, MN, RNCteam led by a clinical nurse specialist recom- OB, Pinnacle Health System, mended and implemented evidence-based prac- Harrisburg, PA tice changes to improve outcomes and decrease readmissions 
Megan L. Strohecker, BSN, RNC-MNN, Pinnacle Health System, Harrisburg, PA

Patricia Maurer, MSN, MBA, RNC-MNN, Pinnacle Health System, Harrisburg, PA

Keywords

hyperbilirubinemia readmission

\section{Newborn Care}

Poster Presentation

Design

The lowa Model of Evidence Based Practice to Promote Quality Care.

\section{Patients/Participants}

Term and late preterm newborns discharged from the newborn nursery and their mothers.

Methods

An interdisciplinary team of hospital and community agencies reviewed literature, examined current practices, and identified strategies for changes to improve outcomes and decrease readmissions for hyperbilirubinemia. Evidencebased practice changes were led by nursing leadership, pediatricians, unit practice committee, and quality committee. Education for nurses, pediatricians, and social workers was essential. Interventions addressed improved risk assessment, breastfeeding support in hospital and after discharge, timely availability of home phototherapy equipment, and establishment of consistent home health follow-up. Collaboration and relationship building between the healthcare system and community agencies were key to our success.

Results

Results included improved risk screening, bilirubin evaluation, and trending. Priority lactation consultant support for at-risk newborns within 24 hours of birth and post-discharge lactation clinic for support and hyperbilirubinemia assessment contributed to improved outcomes. Home phototherapy equipment on the nursing unit through consignment facilitated timely discharge and early initiation/continuity of treatment. Home health networks for newborns provided follow-up assessment and breastfeeding support. Pre-and post-implementation data included risk factors; gestational age; feeding methods, support, and outcomes; discharge and readmission bilirubin levels; home health follow-up referrals; and home phototherapy initiation. None of the newborns having home phototherapy were readmitted. Postimplementation demonstrated a $75 \%$ reduction in readmissions.

\section{Conclusion/Implications for Nursing Practice}

An interdisciplinary approach effectively addressed the risk of newborn hyperbilirubinemia. Because bilirubin levels continue to increase after discharge from the hospital, continued assessment of the newborn that includes physiological data as well as breastfeeding support is essential. Establishing a network of home healthcare providers committed to providing specialized services for at-risk newborns has improved follow-up. Providing home phototherapy equipment before discharge facilitates uninterrupted phototherapy for newborns and reduces family stress. Early initiation of home phototherapy has decreased newborn risk of injury by earlier initiation of treatment, prevented newborn-parent separation through hospitalization, and reduced hospital costs that are unreimbursed by third-party payers. Each of these components has provided seamless care to these vulnerable newborns and has improved outcomes.

\section{Exploration of Bacteria Comprising the Human Skin Microbiome Throughout the First Year of Life}

Kimberly Capone, PhD, Johnson \& Johnson Consumer Companies, Inc., Skillman, NJ

Janeta Nikolovski, PhD, Johnson \& Johnson Consumer Companies, Inc., Skillman, NJ

Georgios N. Stamatas, PhD, Johnson \& Johnson, Sante'

Beaute' France,

Issy-les-Moulineaux

Mark Green, BS, Johnson \& Johnson Consumer Companies, Inc., Skillman, NJ

\section{Objective}

ittle is known about bacteria on infant skin, especially during the postpartum period. Even less is known about the relationship between bacterial colonization, body location, and age. The aim of this study was to learn more about the skin's microbiome immediately after birth and throughout the first year of life.

\section{Design \\ Cross-sectional clinical study.}

\section{Setting}

Routine clinical and laboratory setting in Skillman, New Jersey.

\section{Patients/Participants}

In the first cohort, five mothers and their neonates (healthy; full-term) were enrolled in the study. Two of the mother-neonate pairs also included the fathers. In the second infant cohort, we enrolled 31 healthy White infants who were equally distributed between sex and age group (1-3, 4-6, and 7-12 months old). Five randomly selected mothers were also included in the study.

\section{Methods}

We took skin flora samples from infants, mothers, and fathers using an established swab technique or a cup scrub method and extracted DNA from these samples using a commercially available extraction kit. We analyzed DNA from skin samples using polymerase chain reaction, gel electrophoresis, and a bacterial tag-encoded FLXtitanium amplicon pyrosequencing approach and compared these samples to previously identified DNA markers from bacteria. 


\section{R E S E A R C H}

Proceedings of the 2012 AWHONN Convention

Results

Infant skin sampling revealed variations in bacterial genera by region. On the forehead, the most predominant bacterial genera were Streptococcus, Staphylococcus, and Propionibacterium whereas the arm contained an abundance of Streptococcus, Staphylococcus, and Corynebacterium. Large numbers of Clostridium, Streptococcus, and Ruminococcus were found on the buttocks. Bacterial diversity also varied by age. DNA analysis of infants and caregivers revealed 28 distinct gel electrophoresis banding pattern types of Propionibacterium acnes ( $P$. acnes) isolates. Many gel-banding patterns were identified in mothers and infants; two gel-banding patterns were shared among infants, mothers, and fathers. Though birth type may have influenced the microflora on arms Stephen Cox, PhD, Research and buttocks, birth type did not appear to affect and Testing Laboratory, LLC, microflora on the forehead.

Conclusion/Implications for Nursing Practice Lubbock, TX

Scot E. Dowd, PhD, Research and Testing Laboratory, LLC,

Bacteria comprising the skin microbiome evolve Lubbock, TX after birth throughout the first year of life. We observed vertical transmission of bacteria from caregiver to infant, demonstrating that physical contact may play a role in the development of the microbiome. Understanding the dynamic nature microbiome

of bacteria residing on infant skin may help to elu- microflora cidate the requirements for maintaining normal, healthy skin as well as provide insight into the etiology and pathophysiology of eczema, cellulitis, impetigo, and other infant skin disorders.
Keywords

neonate

skin

\section{Newborn Care}

Poster Presentation

\section{Black Infant Mortality: Community Stories}

\section{Objective}

T he purpose of this qualitative study is to use community stories to identify barriers of prenatal and pediatric health care by members of communities with the highest rates of Black fetal and infant mortality.

\section{Design}

The study is a qualitative descriptive study and is informed by Story Inquiry. The five-inquiry process of that guided the study including the following: gather stories about complicated health issues; begin to decipher the complicating health challenge; describe the developing story plot; identify movement toward resolving; and synthesize findings to address the research question.

\section{Setting}

Housing complexes located in the communities with the highest rates of Black fetal and infant mortality in Broward County, Florida.

\section{Patients/Participants}

Participants were a convenience sample of African American community members who choose to attend discussions in their communities about the health of pregnant women and infants and consented to share their stories.
Methods

Community meetings were held in gathering places within the identified zip codes that have the highest rates of black infant mortality. Institutional Review Board approval was obtained through Florida Atlantic University. Verbal consent statements were read prior to the inquiry, and a written copy was provided to all participants. Sessions were recorded, transcribed, and analyzed. All data were de-identified. The Atlas program was used in the analysis of the qualitative data.

\section{Results}

The themes that emerged from the analysis included access issues to health care, including Medicaid insurance barriers, lack of referral to specific healthcare sources, lack of availability except emergency rooms for after hours health care, and lack of coordination of care.

Conclusion/Implications for Nursing Practice

Identification of barriers to care that may prevent infant mortality assists in multidisciplinary planning to overcome these barriers. Informing at-risk communities of risks to health supports community involvement in solution planning.
Mary Ellen Wright, MSN, ARPN, CPNP, Mission

Hospital System, Asheville, NC

\section{Keywords}

infant mortality

community care

access to care

disparity

\section{Newborn Care}

Poster Presentation 


\section{Mother-Infant Synchrony During Preterm Infant Feeding}

Barbara Reyna, $\mathrm{PhD}, \mathrm{RN}$, NNP-BC, VCU Health System, Children's Hospital of Richmond, Richmond, VA

Rita H. Pickler, PhD, RN, PNP-BC, FAAN, Cincinnati Children's Hospital Medical Center, Cincinnati, $\mathrm{OH}$

Lisa F. Brown, PhD, RN, Virginia Commonwealth University, School of Nursing, Richmond, VA

Keywords mother-infant synchrony mother-infant interaction synchrony tool preterm infant feeding

Newborn Care Poster Presentation

Setting

As part of a larger study examining maternal feeding competence 43 mothers were videotaped while feeding their preterm infants at three intervals: just before discharge in the nursery, and in their home at 1 month and 4 months corrected gestational age.

\section{Patients/Participants}

A convenience sample of 10 mother-infant dyads from the larger data set who completed all three data collection points (30 data subsets) were used for this study.

\section{Methods}

The Noldus Observer XT 8.0 was used for coding and data review. The Maternal-Infant Synchrony Scale was created from pilot data and definitions were further refined. The frequency of occurrence for selected behaviors, the percentage of time behaviors occurred during feeding, and the changes in behaviors over the three observation periods were calculated.

\section{Results}

The synchrony tool developed in this study is one of only a few tools designed to measure synchrony early in the development of the mother-infant relationship. The Maternal-Infant Synchrony Scale demonstrates that changes occur in mother and infant behavior over time. Mothers were attentive and focused during feedings and monitored their infants' sucking intently, but there was little interaction between the dyad. The infant attempted more frequently to interact with the mother than the mother to engage with the infant. The influence of infant maturation on feeding behaviors was evident across observations.

\section{Conclusion/Implications for Nursing Practice}

This study revealed behaviors that are descriptive of the interaction and can be used to develop interventions that would support the developing relationship. Use of the Maternal-Infant Synchrony Scale with a larger sample size and a cohort of healthy, term newborns is needed to establish the Maternal-Infant Synchrony Scale as a valid and reliable measure of synchrony.

\section{Use of a Video-Ethnographic Intervention, PRECESS Immersion Method, to Improve Skin-to-Skin Care and Breastfeeding Rates}

Jeannette T. Crenshaw, DNP, MSN, RN, LCCE, IBCLC, NEA-BC, University of Texas at Arlington, Arlington, TX

Kajsa Hilarie Brimdyr, PhD, CLC, Healthy Children Project, East Sandwich, MA

Jane Dimmitt Champion, $\mathrm{PhD}$, RN, FNP, CNS, FAAN, Texas Tech Health Sciences Center, Lubbock, TX

Richard E. Gilder, RN, BSN, MS, CNOR, BCNI, Baylor University Medial Cetner, Dallas, TX

\section{Objective}

D art 1: To describe the rate of exclusive breastfeeding at hospital discharge in healthy mothers and infants who had immediate and uninterrupted skin-to-skin care after vaginal or cesarean birth during a 5-day intervention (Practice, Reflection, Education and Training immersion method) Part 2: To assess for improvements and sustainability in monthly skin-to-skin and exclusive breastfeeding rates.

\section{Design}

Part 1: Descriptive, observational, nonexperimental with video-ethnography and interactive analysis (Practice, Reflection, Education and Training combined with ethnography for sustainable success) during a 5-day quality improvement pilot research study. Part 2: Electronic medical record review to test for differences in monthly skin-toskin and exclusive breastfeeding rates at hospital discharge after the Practice, Reflection, Education and Training intervention.

Setting

Nonprofit private medical center in the southwest United States with 793 beds and 6,000 births per year. 
Patients/Participants

Part 1: convenience sample of 11 healthy Englishspeaking women $>18$ years, admitted to labor and delivery for vaginal birth or scheduled cesarean. Part 2: electronic medical record review.

\section{Methods}

Part 1: descriptive statistics; Practice, Reflection, Education and Training Immersion Method (August 13-17, 2010); barriers and potential solutions identified by staff; comments about skinto-skin experiences. Part 2: inferential statistics Pearson chi-square to test for significant differences between and among monthly rates of skinto-skin care and exclusive breastfeeding (95\% confidence interval; $p<.05$ ) (baseline, July 2010; post-intervention, August-December, 2010).

Results

Part 1: 10 (91\%) received immediate and 8 (73\%) received uninterrupted skin-to-skin care; $6(67 \%)$ of the infants whose mothers planned to breastwent through all nine instinctive stages dur- RN, FAAN, Texas Health ing skin-to-skin care; 5 (83\%) of the 6 infants who Presbyterian Hospital Dallas, went through all nine stages were discharged as Dallas, TX

exclusively breastfed. Part 2: comparison rates Kristin Svennson, PhD, RN, (Pearson chi-square 23.798, $d f=5$ ) between and MTD, Karolinska Institute, among months showed a significant difference in Stockhom, Sweden month-to-month rates $(p=.000)$

\section{Conclusion/Implications for Nursing Practice}

The Practice, Reflection, Education and Training Immersion Method may rapidly improve skin-toskin care; infants who complete all nine states during skin-to-skin may be more likely to exclusively breastfeed at discharge; mothers need support during skin-to-skin care to recognize their infants' readiness to breastfeed; staff who are educated about infants' nine instinctive stages during skin-to-skin may be more effective at providing evidence-based clinical care after birth skin-toskin care may reduce maternal stress and improve satisfaction, particularly during cesarean surgery.
Ann-Marie Widström, PhD, RN, MTD, Karolinska Institute, Stockholm, Sweden

Karin Cadwell, PhD, RN,

IBCLC, FAAN, ANLC,

Healthy Children Project, East

Sandwich, TX

\section{Keywords}

skin-to-skin care

skin-to-skin contact

nine skin-to-skin stages

exclusive breastfeeding

video-ethnography

reflective practice

evidence-based maternity

practices

PRECESS

\section{Newborn Care \\ Poster Presentation}

\section{Cesarean Mothers' Perception of Benefits Associated With Skin-to-Skin Contact}

\begin{abstract}
Objective
To describe the perceptions of mothers giving birth by cesarean of the benefits of skin-toskin contact (also called Kangaroo Care) with their newborn infants immediately following birth in the labor and delivery unit. Research has identified benefits associated with skin-to-skin contact for new mothers and their infants. Nevertheless, application of this valuable bonding technique varies greatly among hospitals. Direct care nurses observed a dichotomy in the birthing experience of mothers having scheduled cesareans compared to mothers having normal vaginal delivery. Mothers giving birth by cesarean had to wait much longer before they could hold their infants skinto-skin.
\end{abstract}

\section{Design}

A nonexperimental, qualitative research design. Institutional Review Board approval was obtained.

Setting

The research setting for this study is Huntington Hospital, a 408-bed, Magnet-designated community hospital in Long Island, New York.

\section{Patients/Participants}

English-speaking, new mothers, 18 years or older, and having a repeat, elective (nonemergency) cesarean are eligible to participate in this nursing research study. Mothers with newborns requiring the special care unit or transfer out of the hospital will be excluded from participating in the study.

\section{Methods}

Through a collaborative registered nurse/physician patient satisfaction initiative, barriers associated with cesarean and timely performance of skin-to-skin contact were identified and eliminated. Maternal Attachment Theory was used as the conceptual framework of the study. Data were collected using a semistructured interview, which was tape recorded. Data analysis was conducted using Van Manen's phenomenologic method with line-by-line isolation of thematic statements. Essential themes and subthemes emerged that illuminated the experiences of mothers giving birth by cesarean.

Results

Preliminary findings indicate that performing skinto-skin contact immediately following birth by
Cheryl Zauderer, PhD, CNM,

NPP, New York Institute of

Technology, Old Westbury, NY

Susan Goldman, RN, CNS, BC, Huntington Hospital,

Huntington, NY

Keywords

skin-to-skin contact

breastfeeding

bonding

postcesarean

\section{Newborn Care}

\section{Poster Presentation}


cesarean reduced mothers' anxiety regarding infant safety, improved trust/confidence in nurses, and improved satisfaction with the cesarean birthing experience.

Conclusion/Implications for Nursing Practice Information gained from this study should be used to empower nurses working in mother-infant settings to evaluate current practices, promote change, and improve the birthing experiences of all new mothers through the use of skin-to-skin contact and other evidence-based nursing practices.

\section{Effects of Breast and Formula Feedings on Neonatal Abstinence Syndrome}

Ursula A. Pritham, PhD, RN-C, Objective

WHCNP, FNP, Georgia

Southern University,

Statesboro, GA

\section{Keywords}

opioid dependency

pregnancy

methadone

buprenorphine

neonatal abstinence syndrome

\section{Newborn Care}

Poster Presentation

$T$

Design

Setting

Maine. examine the effect of infant feeding methods on neonatal abstinence syndrome.

Retrospective study.

Labor and delivery unit and neonatal intensive care unit, Eastern Maine Medical Center, Bangor,

\section{Patients/Participants}

One hundred fifty-two opioid-dependent women on methadone maintenance therapy $(n=136)$ or buprenorphine maintenance therapy $(n=16)$ during pregnancy and their neonates. The neonates were born between January 1, 2005 and December 31, 2007.

Methods

The researcher reviewed the electronic medical records of all opioid-dependent women who were on methadone maintenance therapy or buprenorphine maintenance therapy when admitted for labor and delivery and their neonates. Infant feeding methods were examined in relation to neonatal abstinence syndrome.
Results

Infant feeding method did not predict neonatal length of stay for neonatal abstinence syndrome; however, there were statistically significant differences between formula- and breastfed infants in relation to the initiation of pharmacologic treatment for neonatal abstinence syndrome. For the group of methadone maintenance therapy or buprenorphine maintenance therapy exposed neonates receiving neonatal abstinence syndrome treatment, those infants who were breastfed began first line therapy with phenobarbital 1.1 day later $(p=.008)$ and their length of stay was shorter by 9.4 days ( $p$ $=.016$ ) as compared to formula-fed neonates or neonates who received formula and breast milk. An analysis of variance with the three infant feeding methods (formula, breast or mixed formula and breast) revealed significant differences in neonatal abstinence syndrome treatment between formulaand breastfed infants but not between the formulafed infants and infants fed formula and breast milk.

Conclusion/Implications for Nursing Practice

Opioid-dependent women on methadone maintenance therapy or buprenorphine maintenance therapy should be encouraged to breastfeed.

\section{Motivational Interviewing to Promote Sustained Breastfeeding With Hispanic Mothers}

Susan Wilhelm, RNC, PhD, UNMC, College of Nursing, Scottsbluff, NE

Keywords breastfeeding Hispanic mothers motivational interviewing

\section{Newborn Care Poster Presentation}

Objective

B reastfeeding rates in Hispanic mothers are dependent on the degree of acculturation. Immigrant mothers breastfeed at a higher rate and duration than Hispanic women born and raised in the United States. Since breastfeeding decreases the risk for obesity, and the prevalence of childhood obesity with its comorbidities of type 2 diabetes and heart disease is highest in this population, finding culturally appropriate interventions to promote sustained breastfeeding is critical. Hispanic mothers are not breastfeeding for the recommended time frame and their infants experience more health disparities. We proposed that the use of a client-centered strategy (motivational interviewing) would promote sustained breastfeeding with a rural cohort of Hispanic mothers 


\section{R E S E A R C H}

Proceedings of the 2012 AWHONN Convention

\section{Design}

An experimental, two-group repeated measures design was used to test the effects of a motivational interviewing client-centered intervention versus an infant safety attention intervention.

Setting

A rural community in the midwest.

Patients/Participants

Fifty-three Hispanic mother-infant dyads were recruited. Of the 53 mother-infant pairs recruited, 18 were available at the 6 -week visit.

Methods

Three key visits were conducted to determine the influence of motivational interviewing on breastfeeding self-efficacy levels; intended length of breastfeeding; and duration of partial breastfeed- ing (measured by maternal self-report, breastfeeding test weights, and oligosaccharide level in infant feces).

Results

Independent $t$ tests and Mann-Whitney $U$ nonparametric tests were used to determine significant group differences. No significant group differences were found for the study variables.

Conclusion/Implications for Nursing Practice

To meet the Healthy People 2020 breastfeeding goals, interventions appropriate for the Hispanic culture must be tested with larger samples. In addition, effective strategies to retain participants in research must be developed. Breastfeeding promotion is the initial health promotion strategy that can decrease acute and chronic illnesses and prevent childhood obesity.

\section{Anxiety in Mothers With Preterm Infants in the Neonatal Intensive Care Unit}

\section{Objective}

To tive synthesize and critically examine qualitative and quantitative research related to mothers' anxiety when their infants were admitted to and discharged from the neonatal intensive care unit (NICU), and interventions neonatal intensive care unit nurses use to alleviate mothers' anxiety while their infants are in neonatal intensive care unit.

\section{Design}

A systematic review of qualitative and quantitative research studies published between 1998 and 2011 was undertaken using the following databases: MEDLINE, PubMed, Cumulative Index to Nursing and Allied Health Literature, Ebscohost, Psychinfo, Science Direct, and OVID.

Setting

A systematic review of qualitative and quantitative research.

Patients/Participants

The search yielded a total of 108 citations, of which 40 articles were identified by title as potentially appropriate; 18 were retained based upon review of their abstracts and meeting inclusion criteria and review purpose.
Methods

Of the 18 studies reviewed, 12 (two mixedmethods, five qualitative, and five quantitative) described mothers' experiences when their preterm infants were in the NICU. Six studies (five quantitative and one qualitative) described nursing interventions to alleviate anxiety of the mothers while infants were in NICU.

\section{Results}

Findings revealed that mothers of preterm infants reported guilt, stress, anxiety, depression, and loss of control during hospitalization of their infants in neonatal intensive care unit. Studies also noted varied nursing interventions to alleviate mothers' anxiety, including massaging, skin-skin contact, or communicating with nurses.

\section{Conclusion/Implications for Nursing Practice}

Further research is needed to evaluate factors affecting changes in mothers' anxiety over time and to compare effectiveness of specific nursing interventions to alleviate mothers' anxiety at the time of discharge of the preterm infant from neonatal intensive care unit. Such research could affect nurses' planning of care and use of interventions to alleviate maternal distress.
Malligamoorthi Jambulingam,

MSN, University of Texas

Medical Branch, Galveston,

Texas, Galveston, TX

Keywords

preterm infants

anxiety of mothers

nursing interventions

systematic review

\section{Newborn Care Poster Presentation}




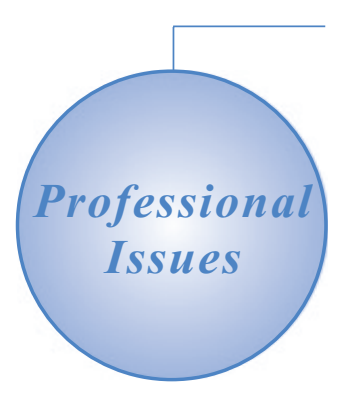

Susan E. Brown Will, MS, RNC, The Johns Hopkins Hospital, Baltimore, MD

Catharine Treanor, MS, RNC, The Johns Hopkins Hospital, Baltimore, MD

Diann L. Snyder, RN, MS, The Johns Hopkins Hospital,

Baltimore, MD

Yvette C. Pugh, BSN, The

Johns Hopkins Hospital,

Baltimore, MD

Corrie Ann McKeen, BSN, RNC, Allina Health Systems, St Paul, MN

Sarah J. Shaefer, RN, PhD, The Johns Hopkins University School of Nursing, Baltimore, MD

Joan Diamond, MSN, RN, The Johns Hopkins Hospital, Baltimore, MD

\section{Keywords} advocacy nurse-patient relationship speaking up

\section{Preventing Obstetric Errors: Building Trust and the Nurse-Patient Partnership}

Objective

To explore patient and nurse expectations for safe care during labor and birth and factors that encourage patients to speak up about care.

\section{Design}

Qualitative study utilizing patient interviews and nurse focus group. Healthy mothers with healthy infants were invited to participate prior to discharge. Family present were interviewed. Experienced labor and birth nurses were invited to participate to elicit perceptions of patients' willingness to speak up about care and nurses' role in this process.

\section{Setting}

Mid-Atlantic level III teaching hospital.

\section{Patients/Participants}

Twenty-three postpartum mothers participated (mean age 25.8, 79\% African American, 26\% married, $63 \% \geq$ high school education). Ten nurses (mean age $40.6,80 \% \geq 6$ years of experience, $80 \%$ White) participated.

\section{Methods}

Interviews continued until saturation was achieved. Questions addressed definition of safe care, comfort in speaking up, and how nurses can encourage patients to speak up.
Focus group and interviews were recorded and transcribed. Researchers reviewed transcripts for themes and completed a computerized theme analysis using Atlas.ti 5. Themes from interviews and focus group were compared and summarized.

\section{Results}

Communication was the theme most frequently identified by patients and nurses. Nurses reported that trust and communication ensure that patients feel comfortable voicing concerns. One nurse encourages patients to use the words concerned, uncomfortable or scared. Lack of communication among the healthcare team was identified. One nurse reported that patients see safety as a healthcare system responsibility and expectation. Some patients identified safety as a shared responsibility relating it is important to "not panic," give all their health history, follow directions, and tell the nurse if something is wrong.

\section{Conclusion/Implications for Nursing Practice}

Visible communication among and between the healthcare team and a patient is critical to make a patient feel safe. "Being there" for a patient helps develop trust and comfort with speaking up. Patients know by the nurse's demeanor whether or not the nurse is there for them. Additional research is needed to further explore these themes, including patients' and nurses' expectations for safe care.

\section{Poster Presentation}

\section{Transitioning From Traditional Maternity Nursing to Family-Centered Maternity Nursing}

Kathleen Kleefisch, DNP, FNP-BC, Purdue University Calumet, Hammond, IN

\section{Keywords}

PARiHS framework

family-centered maternity care

educational modules

maternity nursing

Professional Issues Poster Presentation care, what is the effect of an educational intervention on the staff nurse's knowledge of evidencebased Family-Centered Nursing Care as compared to the knowledge of traditional nursing maternity practices?

search Implementation in Health Services framework, we hypothesized that no practice change would occur unless nurses understand and appreciate the relevance of evidence-based maternity care. A within design was used to address the clinical question, In the transition to FamilyCentered Nursing Care from traditional maternity

\section{Design}

The major steps of the project were to (a) identify new skills and knowledge needed by the staff nurses to function successfully and to integrate the new philosophy of Family-Centered Nursing 


\section{R E S E A R C H}

Care, (b) select through a process of professional consensus the top evidence-based clinical recommendations, (c) design educational modules to increase staff nurses' knowledge of FamilyCentered Nursing Care, and (d) measure knowledge gained, to determine the impact of the educational intervention.

\section{Patients/Participants}

All participants were female staff nurses between the ages of 25 and 65 years, working on the maternity unit: 26 (55.3\%) worked on the nursery unit, 19 $(40.4 \%)$ worked on the postpartum unit, and two $(4.3 \%)$ were managers on the units.

\section{Methods}

Six pre- and post tests were given in online format to assess the participant's knowledge of educational module objectives. Descriptive statistics such as number, mean, and standard deviation was used to analyze demographic data. Paired $t$ test was used to analyze pre- and post test score. A 95\% confidence interval was set $(p=.05)$.

\section{Implementation Strategies}

During a period of 3 months, nine educational sessions were scheduled and completed. An identi- cal format was followed for each session. The four modules were (a) introduction to Family-Centered Nursing Care, (b) postpartum assessment/nursing care, (c) newborn assessment/nursing care, and (d) perinatal safety and risk management.

\section{Results}

The pre- and post test scores of all the educational modules were compared by calculating a paired-samples $t$ test. The total mean score on the pretest was $0.57(S D=0.10)$. The total mean score on post test was $0.90(S D=.065)$. These results exhibited a significant difference in test scores $(t(46)=-28.426, p<.000)$.

Conclusion/Implications for Nursing Practice The provision of expert-facilitated evidence-based education, guided by the Promoting Action on Research Implementation in Health Services framework, which considers evidence, context, and facilitation, can be an impetus for change in healthcare practice. An innovative educational program tailored to the learning needs of experienced staff nurses can contribute to improvement in evidence-based nursing practice and patient care.

\section{Intrapartum Nurses' Experience of Supporting Women Who Anticipate a Preterm Birth}

\begin{abstract}
Objective
T o explore and describe, from the nurses' perspective, the labor support that intrapartum nurses provide to women anticipating a preterm birth.

Design

Qualitative; Husserlian phenomenologic approach.

Setting

Interviews were conducted face-to-face in a private setting of the participants' choice that was comfortable and convenient to each participant.
\end{abstract}

Patients/Participants

A purposive, snowball sampling design was used to identify nurse participants who had experience supporting women anticipating the birth of a preterm infant; 10 intrapartum nurses were recruited. All the nurse participants were female with an age range of 26 to 56 and a mean of 38 years. Seven of the 10 participants (70\%) had a Bachelor of Science degree in Nursing, two participants (20\%) had an Associate's Degree in Nursing, and one participant (10\%) had a Diploma in Nursing. The years of overall nursing experience ranged from 4 to 36 years with a mean of 14. Partici- Suzanne Lundeen, RNC, PhD, pants' years of nursing experience as an intra- Platt College, Aurora, CO partum nurse ranged from 4 to 35 years, with a mean of 12 .

\section{Methods}

Data collection included face-to-face unstructured, open-ended interviews, methodological and personal journals, and field notes. Interviews were audio recorded and transcribed verbatim. Colaizzi's phenomenologic data analysis method was conducted and Lincoln's and Guba's trustworthiness criteria were used to ensure rigor.

\section{Results}

Ten theme clusters emerged from the data: two primary theme clusters and eight supporting subtheme clusters. Primary theme cluster authentically attending had the supporting subthemes of befriending, allowing time and space, interpreting what matters, and intentional shifting. Subtheme clusters of teaching and learning, knowing and not knowing, recreating home, and knowing when to call pediatrician support the primary theme of bridging. 
Conclusion/Implications for Nursing Practice

Knowledge gained from this study has significance for nursing practice, health professionals,

and educators by providing a novel description or essence of the support that intrapartum nurses provide to the high-risk population of women awaiting the birth of a preterm infant.

\section{Perceptions of Nurses Participating in Obstetric Hemorrhage Simulation Training}

Deborah M. Heine, BSN, RNC, Advocate Christ Medical Center, Oak Lawn, IL

Cheryl Lefaiver, $\mathrm{PhD}, \mathrm{RN}$, Advocate Christ Medical Center, Oak Lawn, IL

Sylvia Parker, RNC, Advocate Christ Medical Center, Oak Lawn, IL

Tina Davis-Larkin, MSN, APN, RNC, Advocate Christ Medical Center, Oak Lawn, IL

Keywords

obstetric hemorrhage

focus group

simulation

\section{Professional Issues}

Poster Presentation

\section{Objective}

T o examine nurses' perceptions of participating in obstetric hemorrhage simulation training. Obstetric hemorrhage is a common cause of maternal death in the United States. In 2009, Illinois mandated training for obstetric hemorrhage using multidisciplinary simulation drills as a safe learning environment for healthcare workers. There was limited information how nurses translated this training into practice.

\section{Design}

Qualitative research using focus groups.

Setting

The Women's and Infant's Health Service department of a level III perinatal center in a large midwest medical center.

\section{Patients/Participants}

Participants included nurses working in labor and delivery or family-centered care. Nurses volunteered to participate in one of three focus groups. The sample of convenience included a total of 18 female nurses aged 27 to 53 years, with a range of obstetric experience from 10 months to 31 years. Six of the participants reported they had not experienced an obstetric hemorrhage prior to the training.

Methods

The study was approved by the health system's Institutional Review Board. The need to sign a consent form was waived to ensure confidentiality; participation was considered implied consent. Participants completed a brief demographic questionnaire and were asked predeter- mined questions related to their perceptions of the training and subsequent experience treating obstetric hemorrhage. All focus groups were audiotaped and transcribed verbatim; sessions lasted approximately 1 hour and 45 minutes.

\section{Results}

Five main themes reflected the participant's perceptions of the training: training viewed as valuable, multidimensional approach to training was beneficial, impact on practice, challenges to implementation, and recommendations to sustain learning. Training was viewed as valuable as nurses reported increased preparedness, role clarity, multidisciplinary interactions, and increased confidence. They described benefits of the multidimensional approach, which included lectures, simulation, skills stations, and debriefing sessions; additionally participants reported different perceptions based on the clinical unit worked. Participants described the translation of training as changing their practice including a systematic and organized response, actual practice change, informal teaching, improved teamwork, and improved patient safety. Conversely, participants reported challenges such as inconsistent implementation of training elements, perception of delayed response time, and different policies by nursing unit. Lastly, participants recommended ways learning could be sustained including refreshers, debriefing sessions in practice, and additional practice changes.

Conclusion/Implications for Nursing Practice The findings will be used to modify future training programs for nurses and make changes to clinical care to improve patient outcomes and safety.

\section{What Were They Thinking? Measuring the Breastfeeding Beliefs and Attitudes of Obstetric Nurses}

Sharon Greene-Hughes, RN, BSN, C-EFM, Mount Sinai Hospital, Chicago, IL

\section{Objective}

N ation-wide breastfeeding rates remain low despite a decade of programming on state and local levels. Beliefs and attitudes of nursing obstetric staff may enhance or hinder the success of breastfeeding initiatives due to subtle messaging. The purpose of this work is to describe the impact of an educational intervention on attitudes 
and beliefs regarding breastfeeding to foster a baby-friendly hospital.

\section{Design}

An intervention study on staff attitudes and beliefs with the use of a reliable and valid tool was conducted with nursing staff. Labor and delivery and postpartum nurses from a large midwestern urban hospital responded to the Breastfeeding Beliefs, Bottle Feeding Beliefs, and Breastfeeding Attitudes Attitude Survey at baseline and following intervention. Following the initial survey, staff were provided with a 5-hour educational intervention "Bridges to Breastfeeding" provided by members of the Illinois Department of Health, Special Supplemental Nutrition Program for Women, Infants, and Children program and breastfeeding advocates.

Patients/Participants

One hundred thirty-nine staff nurses.

\section{Methods}

Pre- and post-intervention survey of nurses' beliefs and attitude.

\section{Results}

Descriptive and inferential statistics were used in the analysis with significance determined with a $p$-value of $<.05$. A total of 139 surveys were ana- lyzed. Eighteen stem questions were grouped into Karen Bry, RN, BA, CPHQ, four categories: beliefs and attitudes regarding in- Mount Sinai Hospital, Chicago, fant health, medical concerns, effect on activities IL of daily living, and privacy and intimacy. There were increases in scores in three of the four categories. Statistically significant findings were identified in the medical and maternal convenience sections. Results also demonstrated a difference between breastfeeding beliefs and attitudes among the obstetric staff. Postpartum nurses had three times the overall positive change in scores compared with the labor and delivery respondents.

\section{Conclusion/Implications for Nursing Practice}

Breastfeeding beliefs and attitudes of the surveyed nurses were affected positively following an educational intervention. Key areas were those related to infant health and nutrition, maternal convenience, and the medical effects of alcohol, tobacco, and junk food. There was no measurable impact on questions related to intimacy or privacy indicating that these beliefs and attitudes were not ideally addressed by the educational offering and may be better addressed through sensitivity training. Understanding what nurses were thinking challenged our assumptions, redirected our training efforts, and identified a commonly cited finding: that some barriers to raising breastfeeding rates may be grounded in nurses own beliefs and attitudes.

\section{Implementation of Targeted Medication Education for Improved Patient Satisfaction}

Objective

$D$ atient education regarding postpartum medications was offered inconsistently and utilized hospital-wide educational materials. Dissatisfaction with this was reflected in the Hospital Consumer Assessment of Health Plans Survey (HCAHPS) results and comments received on surveys and post-discharge telephone follow-up. The quality nurse scholar embarked on a project to develop targeted materials and address inconsistent medication education in the postpartum population to increase patient knowledge and improve patient satisfaction scores in the Hospital Consumer Assessment of Health Plans Survey medication domain.

\section{Design}

Patient-centered care, including adequate information regarding medications, positively influences patient outcomes and patient satisfaction. Educational materials should be easily understandable, tailored to the population, and delivered consistently. Utilization of established practices like bedside report and modification Kristine M. Rovell, MSN, RN, of current documentation to support practice C, Riverview Medical Center, change improves accuracy of information pro- Red Bank, NJ vided and imbeds new processes in the care rou- Jennifer B. Bradle, MSN, RN, tine.

CNS, Riverview Medical

Center, Red Bank, NJ

Patients/Participants

Kimberly Kruesi, RN,

All postpartum patients returning the patient satis- Riverview Medical Center, Red faction surveys from November 1, 2009, to November 30, 2010; quality nurse scholar, nurse manager, clinical nurse specialist, clinical pharmacist, postpartum nursing team members.

Bank, NJ

Keywords

HCAHPS

patient education

medication

\section{Methods}

Analysis and comparison of the survey medication domain scores and comments received from November 1, 2009, to May 31, 2010, to those received from June 1, 2010, to November 30, 2010. Quality nurse scholar identified project members, reviewed objective, and developed timeline for implementation. The team identified barriers to achieving identified outcomes and discussed alternative initiatives to improve 
patient education. Team members developed unitspecific medication lists for patient education and enhanced patient card filing system with features to improve nurse-to-nurse communication and encourage continuity of consistent education. All postpartum team members were in-serviced in the objective of the project, use of the educational tools, and revision to the patient card filing system. Project tools were implemented in May 2010.

\section{Results}

The Hospital Consumer Assessment of Health Plans Survey medication domain scores increased from a composite score of $59 \%$ in November 2009 to a composite score of $71 \%$ in November 2010 illustrating a 12\% increase af- ter implementation of the project. Scores showed improvement each month after implementation.

\section{Conclusion/Implications for Nursing Practice}

Implementation of unit-specific medication education tools and enhancement to nurse-to-nurse communication practices affected a significant increase in patient satisfaction scores in the medication domain of the survey insure patients are knowledgeable in all aspects of self-care, including medication administration and side effects. Examination of current educational practices for appropriateness and effectiveness is strongly encouraged especially with the impending reform encouraging accountable care and associating these practices and patient satisfaction with reimbursement.

\section{Development of the Neonatal Resource Nurse Role in the Labor and Delivery Unit: Changing the Way We Support Mothers and Newborns During the Transition Period}

Dixie K. Weber, MS, RNC,
Eastern Idaho Regional Medical
Center (HCA), Idaho Falls, ID
Patricia Anderson, RN, MBA,
Eastern Idaho Regional Medical
Center (HCA), Idaho Falls, ID
Keywords
newborn
transition
care
resuscitation
stabilization
collaboration

Professional Issues Poster Presentation
Objectives

1. Development of best practice outcomes for healthy and critical newborns during the transition period.

2. Analysis and stabilization of the critical newborn from the labor and delivery unit to the neonatal intensive care unit.

3. Increased recognition of signs and symptoms of postpartum complications by the labor and delivery nurse as a result of focusing solely on the mother.

\section{Design}

The Resource Nurse role was developed by evaluating the literature, discussing the current clinical concerns related to the stabilization, and transportation of critical newborns out of the labor and delivery unit to the neonatal intensive care unit, and care issues identified by the pediatric providers. Collaboration between neonatology, obstetrics, pediatrics, and nursing was utilized to ensure all newborn aspects of care during the transition period were considered and a priority of care was determined.

\section{Patients/Participants}

Neonatal and obstetric nurses were the primary clinicians who developed the Resource Nurse role.

Methods

Utilization of literature review, skills assessment and education for resuscitation techniques and normal transition care, collaborative discussions for process flow issues and case reviews were used to refine the care process.

Implementation Strategies

Weekly meetings were initiated during which key players were identified and a timeline was established. Separate taskforce meetings were utilized to create clear clinical pathways for newborn charting, room set up, breastfeeding support, and post-transition period handoff. One-on-one interviews were conducted at the 60-day mark after the role was implemented and will be conducted at the 6-month mark for evaluation of progression in nursing comfort and workflow.

\section{Results}

Results included the following improved patient outcomes related to increased collaboration between neonatology, obstetrics, and pediatric nurses, and providers; decreased rates of hypothermia, hypoglycemia, and inappropriate patient admission to the neonatal intensive care unit. Neonatal and obstetric nursing staff reported an increase in their clinical understanding of newborn physiology during the transition period and an increased comfort with initiating resuscitation for newborns based on neonatal resuscitation guidelines.

\section{Conclusion/Implications for Nursing Practice}

We saw an overall elevation in the level of care provided to newborns and mothers in the labor and delivery setting. We implemented fundamental changes in the clinical approach to the care 


\section{R E S E A R C H}

Proceedings of the 2012 AWHONN Convention

of the newborn by providing the newborn his/her own nurse to monitor and evaluate the complex transition period. Increased communication and collaboration between two specialties resulted and allowed improved planning, implementing, and supporting best outcomes for newborns and mothers.

\section{Consensus Definitions for Perinatal Failure to Rescue: A Delphi Study}

\begin{abstract}
Objective
T he increased focus on patient safety and quality makes it necessary to identify tools that assist the perinatal nursing specialty to measure and modify processes to improve safety and assess quality. Such a tool is perinatal failure to rescue. Prior use of perinatal failure to rescue in research noted difficulty in locating the tool's elements in perinatal documentation. Incorporation of perinatal failure to rescue into electronic documentation systems may improve the ability to retrieve the elements, equate them to corresponding outcomes, and benchmark one facility's outcomes against another. However, to incorporate perinatal failure to rescue elements into electronic systems, standard (consensus) definitions for the elements are necessary. The purpose of this study was to identify consensus definitions for the elements of perinatal failure to rescue.
\end{abstract}

Design

Exploratory study, using a modified Delphi technique.

Setting

The setting was virtual, with participants completing Delphi study rounds through a series of online surveys. Participants were recruited through an online perinatal nursing discussion list with over 800 active members.

\section{Patients/Participants}

Participants included 29 experienced labor and delivery nurses from a variety of practice settings. Experience was defined as at least 5 years current practice in a labor and delivery setting, as well as completion of an Association of Women's Health, Obstetric and Neonatal Nurses interme- diate or advanced fetal heart monitoring course, Catherine Ivory, RN, BC, work as an Association of Women's Health, Ob- MSN, Mountain States Health stetric and Neonatal Nurses fetal heart monitoring Alliance, Johnson City, TN instructor and/or certification in fetal heart monitoring.

\section{Keywords} research

Delphi studies

failure to rescue

Methods

The initial online survey asked participants to list the words they used to document various perinatal failure to rescue elements. Also, since fetal monitoring frequency depends on whether the mother or fetus is considered high risk, participants were asked to identify the maternal and/or fetal characteristics that classified a mother or fetus as either high or low risk. Subsequent rounds (2) summarized responses from the previous rounds with the goal to reach at least $75 \%$ consensus for each perinatal failure to rescue element.

\section{Results}

Of the 70 distinct elements of perinatal failure to rescue, participants reached at least $75 \%$ consensus for 66 (94\%).

Conclusion/Implications for Nursing Practice This study is foundational and supports the need for further research. Study findings may be used to incorporate perinatal failure to rescue into electronic systems in a standard format, perhaps even using standard, coded nursing terminologies. Standardizing the use of perinatal failure to rescue may permit easier correlation of perinatal failure to rescue to patient outcomes and safer perinatal nursing practice. Findings also suggest a framework for determining risk as such risk relates to the frequency of fetal heart monitoring assessment. 


\section{Changing Knowledge and Attitude Toward Maternity Care Practices Consistent With the Baby-Friendly Hospital Initiative}

Marjorie Higgins Young, BSN, Objective

RNC, IBCLC, LewisGale

Hospital Montgomery,

Blacksburg, VA

Keywords

Baby-Friendly Hospital

Initiative

perinatal care measures

Surgeon General

Professional Issues

Poster Presentation

М

ill an educational intervention increase tudes of birthing center staff in regards to maternity-care practices related to the BabyFriendly Hospital Initiative? This intervention will serve as a foundational step to prepare our hospital for Baby-Friendly Hospital Initiative designation.

\section{Design}

This study used a pretest-posttest quasiexperimental design with the target population healthcare practitioners in facilities that provide maternity-care services.

\section{Setting}

The accessible study population consisted of birthing center staff in a rural community hospital.

\section{Patients/Participants}

The study participants consisted of 8 pediatricians, 4 obstetricians, and 28 nurses. Included in the nurses' (possessive) group were three nursing students and four obstetric technicians.

\section{Methods}

The pre- and post- test knowledge/attitude survey tool was an investigator-developed tool. The survey tool items were developed in accordance with the Baby-Friendly Hospital Initiative "10 Steps to Successful Breastfeeding." Content validity of the survey tool was verified by expert staff from the Baby-Friendly Hospital Initiative. A pairedsamples $t$ test using pre- and post test survey results was conducted on each individual survey item to evaluate the impact of the educational intervention on the understanding of and attitudes about maternity care practices consistent with the Baby-Friendly Hospital Initiative. The eta-squared statistics were calculated for effect size.

\section{Results}

On the survey's 10 knowledge questions, the average overall pretest score was $76 \%$. This increased to $95 \%$ following the staff training. Of these 10 questions, further analysis demonstrated that five of the questions did not meet criteria for statistical significance when calculated separately but when combined had a pretest score of $97 \%$ and a post test score of $98.5 \%$. On questions $3,6,7$, 8 , and 9 , the percentage correct was high on preand post tests indicating either foreknowledge or a simplistic question. The remaining five questions demonstrated strong statistical significance with a combined mean $p<.01$ indicating the effectiveness of the training on the participants knowledge. On the Likert scale questions concerning attitude with 1 representing strongly disagree and 5 representing strongly agree, the pretest mean score was 3.84 , increasing to 4.53 following the intervention. This increase carries a $p<.0012$, indicating a strongly significant increase in positive attitude toward Baby-Friendly Hospital Initiative maternity care practices.

Conclusion/Implications for Nursing Practice

Our findings indicate a significant impact on knowledge and attitude about Baby-Friendly Hospital Initiative consistent maternity-care practices when healthcare staff is provided with targeted education.

\section{The Obstetric Registered Nurse's Lived Experience of Birth Trauma}

Jennifer M. Scotese Baxter, $\mathrm{RN}$, University of Illinois at Chicago, New York, NY

\begin{abstract}
Objective
To explore the obstetric nurse's lived experience with birth trauma. In general, nurses exposed to trauma during the course of their work or who care for traumatized patients are often at risk for mental, physical, and emotional problems, but little research about this phenomenon exists specific to the obstetric nurse population. Birth trauma has been defined as an event during the labor and
\end{abstract}

delivery process involving an actual or threatened serious injury or death to the mother or her infant. Events can range from pain during labor to fetal or maternal death. Exposure to such trauma may have a significant effect on obstetric nurses and their ability to care for birthing women. By exploring the experience of trauma from the perspective of obstetric nurses, we can identify ways to improve the hospital environment for obstetric 
nurses, birthing women, and others involved in the birthing process.

\section{Design}

A qualitative, hermeneutical phenomenologic design.

\section{Setting}

Four participants were recruited and interviewed in Chicago, IL.

\section{Patients/Participants}

Eligible participants (a) worked full-time in an obstetric environment of a hospital for at least 1 year within the last 5 years; (b) had an experience with birth trauma while working in such a setting; (c) speak, read, and write in English; and (d) were able to participate in a face-to-face interview in the Chicago area. Pregnant women were excluded from this study.

\section{Methods}

After obtaining informed consent, each subject was interviewed and asked to describe in detail one or more birth trauma events she experienced while caring for a woman during the birthing process. The interviews were digitally recorded and transcribed as were the researcher's own postinterview summary notes.

Results

Analysis revealed themes surrounding the experience of birth trauma among the participants such as "It shapes who you are," which reflects how nurses are changed personally and professionally from their trauma experiences. The themes illustrate immediate and long-term meanings of what it is like to be an obstetric nurse affected by trauma.

Conclusion/Implications for Nursing Practice

Though future studies are needed to explore a broader definition of trauma experiences for obstetric nurses, these results will challenge researchers, hospital management, nurse educators, and nurses themselves to consider the personal and professional implications of trauma exposure in obstetric nursing practice.

\section{The Obstetric Nursing Self-Efficacy Scale: A Report of Initial Reliability and Validity Data for a New Instrument}

Objective

Tr he he Obstetric Nursing Self-Efficacy instrument was developed to measure perception of selfefficacy related to the ability to provide safe obstetric nursing care among nursing students after the completion of an obstetric clinical rotation. The purpose of this poster is to report promising preliminary findings related to the reliability of the Obstetric Nursing Self-Efficacy instrument and encourage psychometric testing of the instrument in other populations.

\section{Design}

Pretesting of the instrument established preliminary reliability and validity prior to deployment in the main study. The instrument was administered to students before and after a 45-hour obstetric clinical rotation to measure perception of self-efficacy.

Setting

The study was completed at a large, southern, public university.

\section{Patients/Participants}

Junior level nursing students who were enrolled in an obstetric theory and clinical course completed the instrument.
Methods

Mary E. Guimond, $\mathrm{PhD}$,

The Obstetric Nursing Self-Efficacy is an 18- WHNP-BC, Duquesne item scale designed to measure perceived selfefficacy in providing safe obstetric nursing care. Bandura's social-cognitive theory was used as the theoretical framework to guide instrument design and content validity was subject to multiple rounds of expert review. Convenience samples for Pretest $1(N=20)$ and Pretest $2(N=46)$ were used; the main study was a randomized cluster design $(N=110)$ to compare differences in learning outcomes between students who completed a standard clinical and those who completed a simulation-enhanced clinical rotation.

\section{Professional Issues}

Poster Presentation

\section{Results}

At the end of 45 hours of obstetric clinical, students completed the Obstetric Nursing SelfEfficacy Scale (Pretest 1, $N=20$ ), split-half reliability scores were calculated at .85 and .96. In Pretest 2, the Obstetric Nursing Self-Efficacy was administered pre- and post-obstetric simulated clinical experience $(N=46)$ alpha $=.92$; validity for the instrument was assumed as selfefficacy scores improved after exposure to simulation. For the main study, alphas of .96 for the pretest and .93 for the test were obtained for the whole group. Though no between-group differences (simulation-enhanced versus standard 
clinical) were detected, self-efficacy scores improved for both groups.

Conclusion/Implications for Nursing Practice The Obstetric Nursing Self-Efficacy instrument has demonstrated good initial reliability and is promising as a valid instrument to measure self-efficacy in obstetric nursing care. Because the Obstetric
Nursing Self-Efficacy is designed to detect behaviors related to safe and effective care in obstetric nursing, it may be well suited as a measure to detect perception of self-efficacy in new nurses prior to and after orientation to the obstetric clinical unit. Further testing in this population is needed to establish its usefulness for this group.

\section{An Innovative Approach to Increasing the Cultural Competence of Nurses Who Care for Childbearing Women and Newborns}

Ella Thomas Heitzler, $\mathrm{PhD}$, WHNP-BC, RNC-OB, Bon Secours Memorial College of Nursing, Richmond, VA

Keywords cultural competence continuing education women's health obstetric nursing neonatal nursing

Professional Issues Poster Presentation
Objective

ignificant health disparities exist between $\checkmark$ childbearing women and newborns from different cultures. Culturally competent nurses are essential to providing adequate care and reducing these disparities. Unfortunately, many nurses acknowledge they are not culturally competent. This study's purpose was to evaluate the effect of two different online cultural competence continuing education interventions on the cultural competence level of obstetric and neonatal nurses. It was hypothesized that there would be a greater increase in the Cultural Competence Assessment scores of the nurses in the socially interactive course group compared to those in the socially isolated course group, and that both groups would have greater increases in Cultural Competence Assessment scores than the control group. The relationships between Cultural Competence Assessment scores and educational level and between Cultural Competence Assessment scores and previous diversity training were also explored.

Design

Experimental pre- and post test.

Setting

The study was conducted online.

Patients/Participants

A nonprobability national sample of obstetric and neonatal nurses.

Methods

Association of Women's Health, Obstetric, and Neonatal Nurses section leaders were asked to distribute information about the study to their membership. Members wishing to participate registered online and were randomly assigned to the three groups. All participants were asked to complete a pretest during the week experimental groups began the 4-week, 10-hour, cultural competence continuing education courses. They were also asked to complete a post test during the final week.

\section{Results}

Ninety-three nurses completed the study. Analysis of covariance revealed a significant difference between groups in post test Cultural Competence Assessment scores after controlling for pretest scores $[F(2,61)=5.2, p=.008]$. The socially isolated group scored significantly higher than the control ( $p=.006)$, but the socially interactive group did not score significantly higher than the control group or the socially isolated group. Cultural Competence Assessment scores were not related to educational level or previous diversity training (yes/no), but were related to number of types of previous diversity training $\left(r_{2}=.173, p=\right.$ .047).

Conclusion/Implications for Nursing Practice Nurses who provide care to childbearing women and newborns are not currently culturally competent. It is recommended that nursing utilize asynchronous socially isolated online continuing education and educate nurses about its technology. Nurses should be exposed to more types of cultural diversity training and must consistently utilize cultural assessment tools, ideally tailored to their specialty area. Finally, nursing areas must have cultural reference materials specific to their patient populations. 


\section{Starting Up: Challenges and Strengths of Beginning a Statewide Home Visiting Program for Pregnant/Parenting Adolescents}

\begin{abstract}
Objective
To describe the experience of nurses implementing an evidence-based home visitation program (Nurse Family Partnership) in six different areas of one state. The Nurse Family Partnership is a home visitation program serving first-time pregnant and parenting adolescents.
\end{abstract}

\section{Design}

This secondary analysis was part of a study assessing the systematic statewide implementation of an evidence-based home visitation program in South Carolina.

\section{Setting}

Six counties in the state of South Carolina.

\section{Patients/Participants}

This report describes findings from in-depth, semistructured interviews of 15 nurses. Fourteen of the 15 participants were women; $50 \%$ had a graduate degree, and mean age was 50.6 (range $=29-65$ years). Nurses had lived in their communities for at least 7 years.

\section{Methods}

The interview guide was derived from the conceptual model of the larger study and addressed the nurse's perceptions of program implementa- Mary Ellen Wright, MSN, tion, collaborative processes, patient needs, and ARPN, CPNP, Mission model fidelity. All interviews were taped and tran- Hospital System, Asheville, NC scribed verbatim. Transcripts were reviewed by two independent reviewers with a third reviewer Keywords assisting with any discrepancies. home visiting

adolescent pregnancy

evidence-based program

Results

Two broad themes emerged: first, preparation for implementation implementation with subthemes of training, motivation, model fidelity, tools for the field, and role evolution; second, challenges in the field with subthemes of patient needs, referrals, recruitment, and nurse-patient relationship. Of note, many participants identified an altruistic motivation for involvement in the evidence-based home visitation program, a need for understanding the scope of model fidelity and having a supportive system.

Conclusion/Implications for Nursing Practice This study adds to the growing body of knowledge on the translation of evidence-based home visitation programs into practice. A systematic understanding of the experiences of nurses during the implementation process can play a key part in ensuring fidelity of a program to the model from the outset. 